\title{
Sensory nerves regulate mesenchymal stromal cell lineage commitment by tuning sympathetic tones
}

\author{
Bo Hu, ${ }^{1,2}$ Xiao Lv, ${ }^{1,3}$ Hao Chen, ${ }^{1}$ Peng Xue, ${ }^{1}$ Bo Gao, ${ }^{1}$ Xiao Wang, ${ }^{1}$ Gehua Zhen, ${ }^{1}$ Janet L. Crane,, Dayu Pan, ${ }^{1}$ Shen Liu, ${ }^{1}$ Shuangfei Ni, ${ }^{1}$ \\ Panfeng Wu, ${ }^{1}$ Weiping Su, ${ }^{1}$ Xiaonan Liu, ${ }^{1}$ Zemin Ling, ${ }^{1}$ Mi Yang, ${ }^{1}$ Ruoxian Deng, ${ }^{1}$ Yusheng Li, ${ }^{1}$ Lei Wang, ${ }^{1}$ Ying Zhang, ${ }^{2}$ Mei Wan, ${ }^{1}$ \\ Zengwu Shao, ${ }^{3}$ Huajiang Chen, ${ }^{2}$ Wen Yuan, ${ }^{2}$ and Xu Cao ${ }^{1}$
}

'Department of Orthopaedic Surgery, Johns Hopkins University, Baltimore, Maryland, USA. ${ }^{2}$ Section of Spine Surgery, Department of Orthopaedics, Changzheng Hospital, Second Military Medical University, Shanghai, China. ${ }^{3}$ Department of Orthopaedics, Union Hospital, Tongji Medical College, Huazhong University of Science and Technology, Wuhan, China.

\begin{abstract}
The sensory nerve was recently identified as being involved in regulation of bone mass accrual. We previously discovered that prostaglandin $E_{2}$ (PCE2) secreted by osteoblasts could activate sensory nerve EP4 receptor to promote bone formation by inhibiting sympathetic activity. However, the fundamental units of bone formation are active osteoblasts, which originate from mesenchymal stromal/stem cells (MSCs). Here, we found that after sensory denervation, knockout of the EP4 receptor in sensory nerves, or knockout of COX-2 in osteoblasts, could significantly promote adipogenesis and inhibit osteogenesis in adult mice. Furthermore, injection of SW033291 (a small molecule that locally increases the PCE2 level) or propranolol (a beta blocker) significantly promoted osteogenesis and inhibited adipogenesis. This effect of SW033291, but not propranolol, was abolished in conditional EP4-KO mice under normal conditions or in the bone repair process. We conclude that the PGE2/EP4 sensory nerve axis could regulate MSC differentiation in bone marrow of adult mice.
\end{abstract}

\section{Introduction}

Sensory nerves are innervated in various organs and tissues, such as skin, lung, kidney, liver, and bone. These nerves sense stimuli such as pain, itch, temperature, taste, and odor $(1,2)$. The function of sensory nerves is essential for collecting information from both internal and external environments, helping individuals adapt to their surroundings and protect themselves from threats (3). Recent evidence has shown that sensory nerves also participate in inflammation, immunity, hematopoiesis, and bone metabolism $(4,5)$. Fukuda et al. first reported that loss of sensory nerves could impair bone mass accrual (5). Conversely, Takeda et al. found that bone metabolism was regulated by the sympathetic nerve system (6). Our previous report showed that sensory nerves could detect the bone-forming "signal" from osteoblasts and further tune down sympathetic activity via the central nervous system, thus controlling bone homeostasis (7).

The abovementioned bone-forming signal is prostaglandin $\mathrm{E}_{2}$ (PGE2). We find that PGE2 activates the EP4 receptor in sensory nerves to promote bone formation via the central nervous system (7). PGE2 is synthesized by COX, is degraded by the enzyme 15hydroxyprostaglandin dehydrogenase $(15-\mathrm{PGDH})(8,9)$, and is well known for its roles in inflammation and pain induction $(8,10$, 11). PGE2 also potently induced bone formation, promoted tissue regeneration, and facilitated bone repair $(12,13)$. Direct injection

Authorship note: BH and X. Lv are co-first authors.

Conflict of interest: The authors have declared that no conflict of interest exists. Copyright: () 2020, American Society for Clinical Investigation.

Submitted: July 8, 2019; Accepted: March 11, 2020; Published: May 26, 2020.

Reference information: J Clin Invest. 2020;130(7):3483-3498.

https://doi.org/10.1172/JCl131554. of PGE2 failed as a treatment for osteoporosis and did not increase bone mass in mice (14). This may be due to the breadth of PGE2's targets, which could have adverse effects. However, a small molecule, SW033291, developed by Zhang et al., may inhibit 15-PGDH activity, thus indirectly increasing PGE2 accumulation in certain microenvironments (12). The group also showed that after injury, SW033291 could boost tissue regeneration in liver and intestine, and promote hematopoiesis to a marked degree (12). Our group also showed that SW033291 injection could directly increase bone mass and promote bone regeneration via the EP4 sensory nerve axis in mice (7). However, we identified the upstream "regulator" but not the downstream "effector" of this axis.

The adult skeleton is continuously being formed and resorbed, starting in utero and continuing throughout adulthood (15). This skeletal remodeling is accomplished by precise coordination between resorptive osteoclasts and bone-forming osteoblasts $(16,17)$. The sequence in bone remodeling cycles starts with osteoclastic bone resorption, followed by osteoblastic bone formation (18), which relies on osteoblast differentiation of constant supplies of bone marrow-derived mesenchymal stromal/ stem cells (MSCs) $(19,20)$. Bone marrow MSCs, a subset of mesenchymal cells, can self-renew and differentiate into multiple lineages to produce adipocytes, osteoblasts, and chondrocytes in bone tissue (21-24). MSCs reside primarily in their bone marrow niche in a quiescent state and become active when the niche is stimulated by factors such as injury, inflammation, and medicine intake (25-27). The MSC niche and microenvironment determine the cells' commitment and self-renewal for bone homeostasis. An impaired niche or microenvironment alters MSC commitment, which has been reported in human diseases such as tissue fibrosis, malignant hematopoiesis, and osteoporosis (28-30). Recent 
A

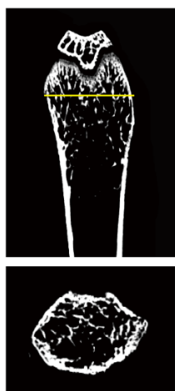

$\operatorname{Trk} A^{\text {wt }}$

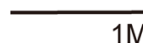

$\mathbf{E}$

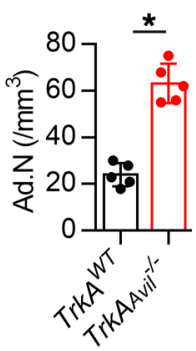

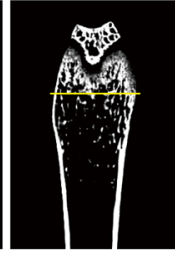

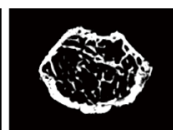

$\operatorname{Trk} A_{A v}$

$\mathbf{F}$

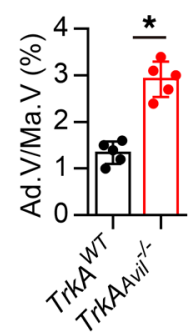

B
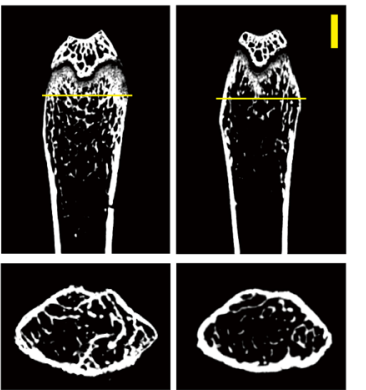

$\operatorname{Trk} A^{w t}$

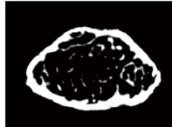

$\operatorname{TrkA}_{\text {Avil }}{ }^{-1}$

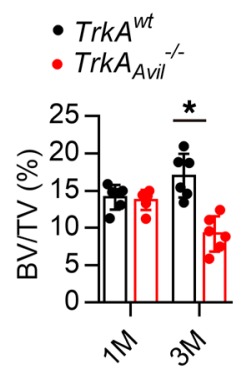

C

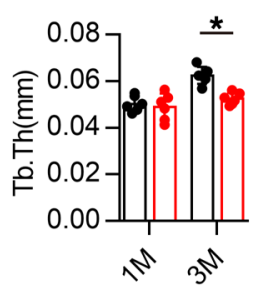

D

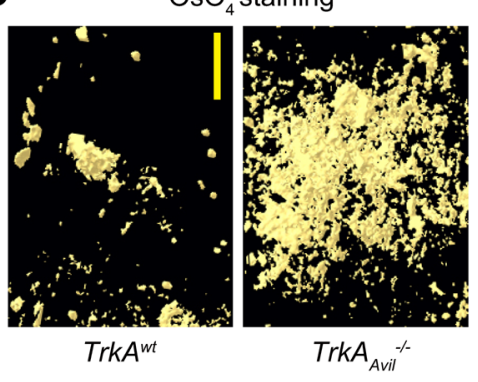

Figure 1. Sensory nerve denervation induces adipogenesis of MSCs at the expense of osteogenesis. (A-C) Representative $\mu$ CT images of femurs from 1- and 3-month-old male TrkA ${ }^{W T}$ and $T r k A_{\text {Avil }}{ }^{-/-}$mice. The thin yellow lines indicate the area where the cross-section images were captured ( $0.5 \mathrm{~mm}$ proximal from the growth plate). Quantitative analysis of Tb.BV/TV and Tb.Th. Scale bar: $1 \mathrm{~mm}$. (D-F) Representative $\mu$ CT-detected OsO $\mathrm{O}_{4}$-stained images of decalcified femurs and quantitative analysis of Ad.N and Ad.V/Ma.V in distal femurs from 3-month-old male TrkA $A^{w T}$ and Trk $A_{\text {Avil }}{ }^{-1-}$ mice. Scale bar: $500 \mu \mathrm{m}$. (G-I) Representative images of immunofluorescence staining and quantitative analysis of the perilipin- (red) and OCN-stained (green) femurs from 3-month-old male Trk $A^{W T}$ and $\operatorname{TrkA}_{\text {Avil }}{ }^{-1-}$ mice. Scale bar: $50 \mu \mathrm{m} . n \geq 6$ per group; ${ }^{*} P<0.05$ (Student's $t$ test). N.Ob./B.Pm, number of osteoblasts per trabecular bone surface.

studies have found that sympathetic nerves could serve as an MSC niche component in bone marrow and regulate interactions between MSCs and hematopoietic stem cells, thus maintaining normal hemopoiesis $(31,32)$.

In our study, we investigated whether the EP4/PGE2 axis in sensory nerves regulates bone mass accrual through direct control of commitment of MSCs by tuning down sympathetic nerve activity. We show that adipogenesis was significantly increased, whereas osteogenesis was markedly decreased in a sensory denervation mouse model, in sensory nerve EP4-KO mice, and in osteoblast COX-2-KO mice. Elevation of PGE2 by SW033291 inhibited adipogenesis and promoted osteogenesis, bone regeneration, and bone fracture healing in WT mice, but not in the KO mouse models. We conclude that MSC differentiation is regulated by the PGE2/EP4 sensory nerve axis in adult mice.

\section{Results}

Sensory nerve denervation induces adipogenesis at the expense of osteogenesis in adult mice. To create a sensory nerve denervation model, we crossed Advillin-Cre mice with nerve growth factor receptor $\operatorname{Trk} A$-floxed $\left(\operatorname{Trk} A^{W T}\right)$ mice to generate $\operatorname{Trk} A_{A v i l}{ }^{-/-}$mice according to our previous work (7). Bone volume fraction (BV/TV) and trabecular thickness (Tb.Th) significantly decreased in 3-month-old $\operatorname{Trk} A_{A v i l^{-/}}$mice relative to WT littermates, whereas no significant changes in these parameters were observed in 1-month-old $\operatorname{Trk} A_{\text {Avil }}{ }^{-/-}$mice (Figure 1, A-C, and Supplemental Figure 1, A-C; supplemental material available online with this article; https:// doi.org/10.1172/JCI131554DS1), suggesting sensory nerve regulation of bone homeostasis, primarily in adult mice. Fat levels were significantly increased in bone marrow of 3-month-old $\operatorname{TrkA}_{A v i l}{ }^{-/-}$ mice compared with WT controls, as evidenced by the markedly higher number of adipocytes and fat droplets in decalcified femurs stained with osmium tetroxide $\left(\mathrm{OsO}_{4}\right)$ (Figure $\left.1, \mathrm{D}-\mathrm{F}\right)$. Immunostaining with osteocalcin (OCN) for osteogenesis and with perilipin for adipogenesis showed a significant increase in the number of adipocytes and decrease in the number of osteoblasts in 3-monthold Trk $A_{A v i l}^{-1-}$ mice relative to WT littermates (Figure 1, G-I). CFU -fibroblast (CFU-F), -adipocyte (CFU-AD), and -osteoblast (CFU-OB) assays with bone marrow cells showed a significant increase in adipogenesis and decreases in osteogenesis and CFU-Fs (Figure 2, A-D) in TrkA ${ }_{\text {Avil }}^{-1-}$ mice relative to WT littermates. Consistent with these results, bone marrow cell expression of the adipogenesis markers Ppary, CCAAT/enhancer-binding protein $\alpha$ (Cebpa), and fatty acid-binding protein 4 (Fabp4) increased significantly, whereas expression of the osteogenesis markers alkaline phosphatase (Alp), collagen type Ia (Col1a1), and Runt-related transcription factor 2 (Runx2) decreased (Supplemental Figure 1, D and E).

These results prompted us to test whether this alteration is caused by sensory nerve regulation of MSCs. Flow cytometry analysis showed that $\mathrm{CD} 45^{-} \mathrm{CD} 31^{-} \mathrm{Sca}-1^{+} \mathrm{CD} 24^{-}$adipogenic progenitor cell (APC) frequency significantly increased, and CD45$\mathrm{CD}^{-} 1^{-} \mathrm{Sca}-1^{-} \mathrm{PDGFR} \alpha^{+}\left(\mathrm{Pa}^{+}\right)$osteogenic progenitor cell (OPC) 
A

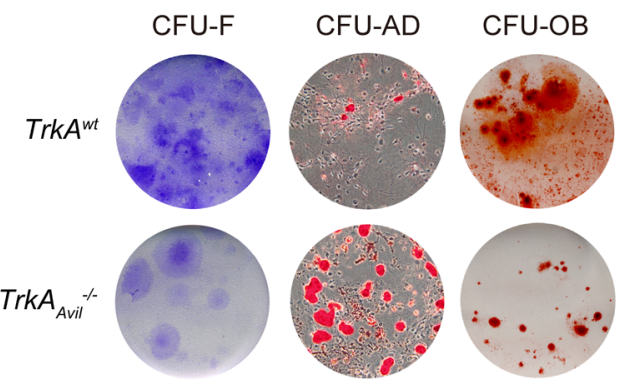

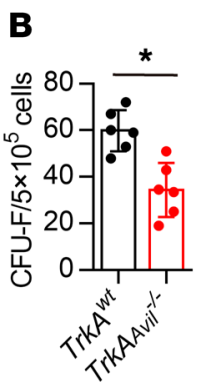

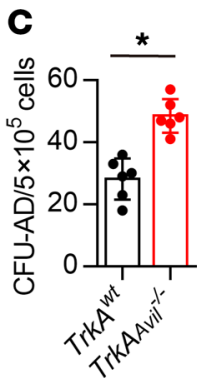

$\mathbf{F}$

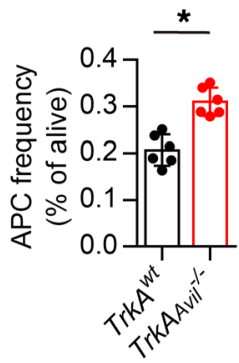

D

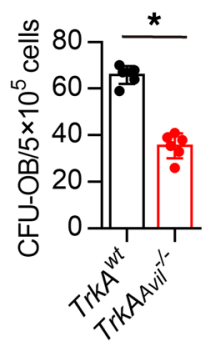

G

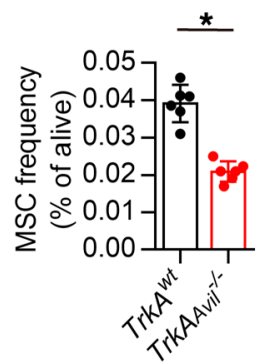

E

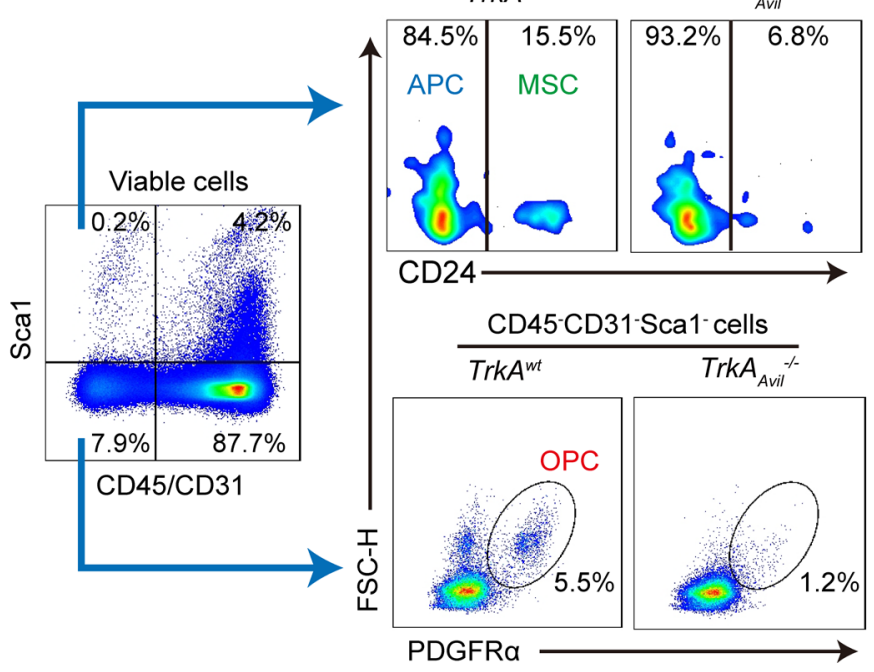

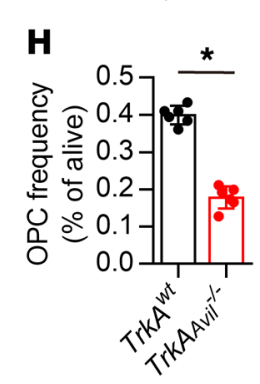

$\frac{\text { CD45-CD31-Sca1 }{ }^{+} \text {cells }}{\operatorname{TrkA}^{\mathrm{wt}}}$

Figure 2. MSCs derived from sensory denervation mice that had undergone adipogenesis. (A-D) Representative images of crystal violet-stained CFU-F, oil red 0 -stained CFU-AD, and alizarin red S-stained CFU-OB. Quantitative analysis of CFU-F, CFU-AD, and CFU-OB MSCs isolated from 3-month-old male TrkA ${ }^{W T}$ and Trk $A_{\text {Avil }}{ }^{-1-}$ mice. (E-H) Representative dot plots of flow cytometry and quantitative analysis of CD45-CD31-Sca-1+CD24- $A P C s$, CD45-CD31-Sca-1 $^{-}$ $\operatorname{PDCFR} \alpha^{+}\left(\mathrm{P}^{+}\right)$OPCs, and CD45-CD31-Sca-1+CD24+ MSCs of live cells isolated from femurs of 3-month-old male Trk $A^{W T}$ and Trk $A_{\text {Avil }}{ }^{-1-}$ mice. $n \geq 6$ per group; ${ }^{*} P<0.05$ (Student's $t$ test).

frequency decreased (Figure 2, E, F, and H). Importantly, the population of $\mathrm{CD} 45^{-} \mathrm{CD} 31^{-} \mathrm{Sca}-1^{+} \mathrm{CD} 24^{+} \mathrm{MSC}$ also decreased (Figure 2, $\mathrm{E}$ and $\mathrm{G}$ ), consistent with the CFU-F results (Figure 2A). Moreover, $\mathrm{BrdU}^{+} \mathrm{OPC}$ and $\mathrm{BrdU}^{+} \mathrm{MSC}$ levels decreased significantly in 3-month-old $\operatorname{TrkA}_{\text {Avil }}{ }^{-1}$ mice relative to WT littermates, whereas $\mathrm{BrdU}^{+}$APCs remained unchanged (Supplemental Figure 2, A-D), consistent with the relative CFU-F levels in the sorted cell population (Supplemental Figure 3, A-C). Also, sorted MSCs showed increased adipogenic potential and decreased osteogenic capacity in $\operatorname{TrkA}_{A v i l}{ }^{-1-}$ mice relative to controls (Supplemental Figure 3, $\mathrm{D}-\mathrm{M})$. These data together suggest that sensory denervation led to increased bone marrow adipogenesis activity in adult mice. Similar results were observed in an inducible sensory denervation model, in which Advillin-Cre mice were crossed with $i D T R^{W T}$ mice to produce $i D T R_{A v i}^{f l-}$ mice according to the methods used in our previous study (7). The results showed that fat levels were significantly increased in the bone marrow of $i D T R_{\text {Avil }}^{f / /}$ mice 4 weeks after injection of diphtheria toxin (DTX) relative to vehicle-treated mice, as evidenced by the markedly higher levels of adipocytes and fat droplets in decalcified femurs stained with $\mathrm{OsO}_{4}$ (Figure 3,
$\mathrm{A}-\mathrm{C})$. The frequency of MSCs and OPCs decreased, whereas the frequency of APCs increased after DTX relative to vehicle treatment in flow cytometry analysis (Figure 3, D-G). Immunostaining of $\mathrm{OCN}$ with perilipin also revealed a significant increase in adipocytes and decrease in osteoblasts after DTX treatment (Figure 3, $\mathrm{H}-\mathrm{J})$. Taken together, these data demonstrate that sensory nerves are essential for maintaining MSCs and balancing their commitment between osteoblast and adipocyte.

Deletion of the EP4 receptor in sensory nerves promotes adipogenesis and inhibits osteogenesis in adult mice. We have shown that the EP4 receptor in sensory nerves is essential for maintaining normal bone mass in adult mice (7). EP4-KO (EP4 $\left.{ }_{\text {Avi }}{ }^{-1}\right)$ mice showed significant decreases in BV/TV and Tb.Th relative to WT littermates (Figure 4, A-C, and Supplemental Figure 4, $A-C)$. Fat levels were significantly increased in bone marrow of $E P 4_{A v i}{ }^{-1-}$ mice relative to WT controls, as evidenced by a markedly higher number of adipocytes and fat droplets in decalcified femurs stained with $\mathrm{OsO}_{4}$ (Figure 4, D-F). To test whether differentiation of MSCs could also be regulated by the EP4 receptor in sensory nerves, we injected the 15-PGDH inhibitor SW033291 
A

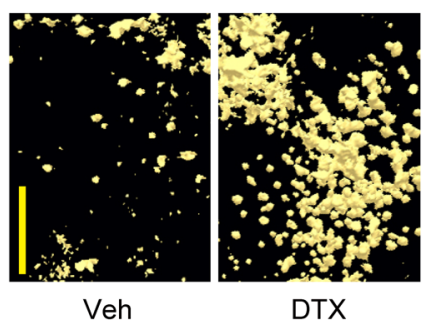

B

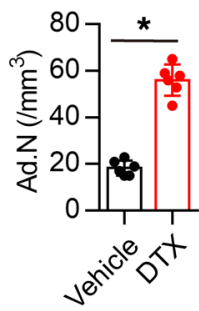

C

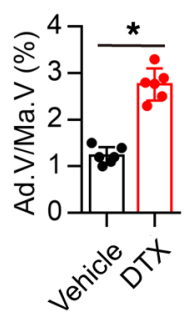

E

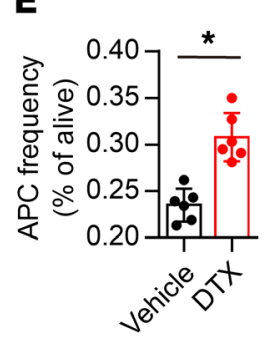

$\mathbf{F}$

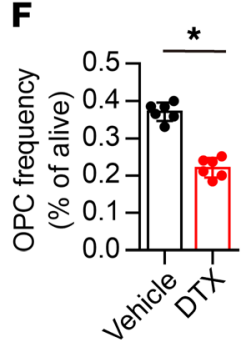

G

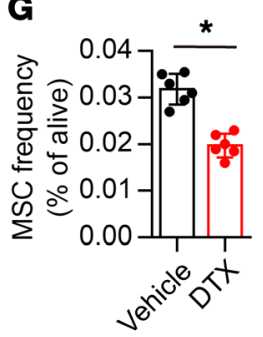

D

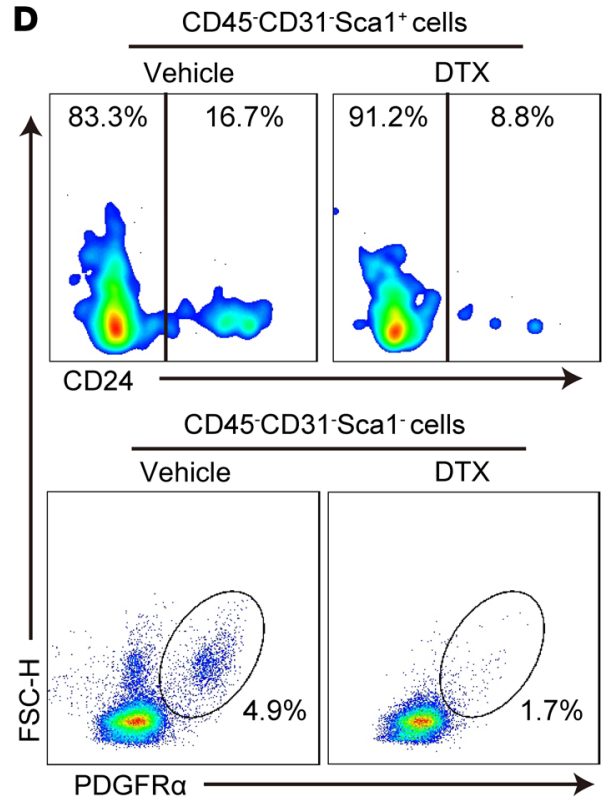

H
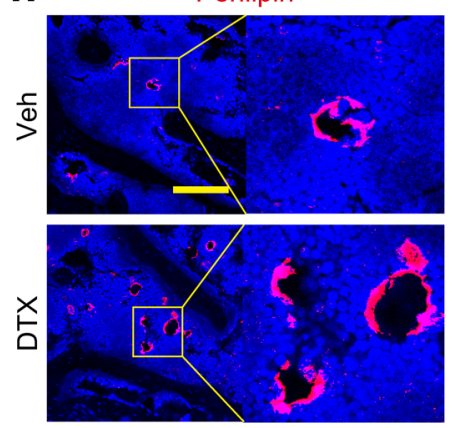
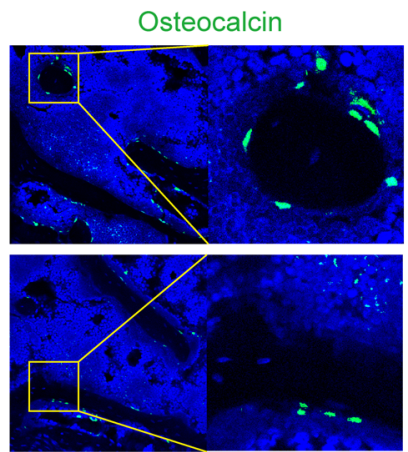

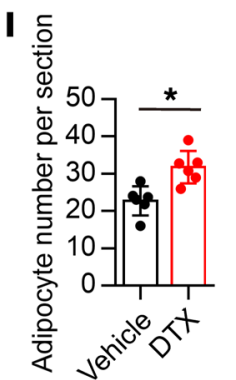

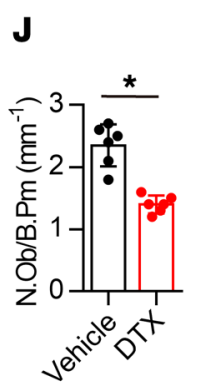

Figure 3. Loss of sensory nerves potentiate adipogenesis. (A-C) Representative $\mu$ CT-detected $\mathrm{OsO}_{4}$-stained images of decalcified femurs and quantitative analysis of Ad.N and Ad.V/Ma.V in distal femurs of 3-month-old male $i D T R_{\text {Avil }}{ }^{+/-}$mice injected with $1 \mu \mathrm{g} / \mathrm{kg} / \mathrm{d}$ vehicle or DTX 3 times a week for 4 consecutive weeks. Scale bar: $500 \mu \mathrm{m}$. (D-G) Representative dot plot images of flow cytometry and quantitative analysis of CD45-CD31-Sca-1+CD24- APCs, CD45-CD31Sca-1-PDCFR $\alpha^{+}\left(\mathrm{P \alpha}^{+}\right)$OPCs, and CD45-CD31-Sca-1+CD24+ MSCs of live cells isolated from femurs of 3-month-old iDTR ${ }_{\text {Avil }}^{+/-}$mice injected with vehicle or DTX for 4 weeks. (H-J) Representative images of immunofluorescence staining and quantitative analysis of perilipin (red) and OCN (green) in femurs from 3-monthold male $i D T R_{\text {Avil }}{ }^{+/-}$mice injected with vehicle (Veh) or DTX for 4 weeks. Scale bar: $50 \mu \mathrm{m}$. $n \geq 6$ per group: ${ }^{*} P<0.05$ (Student's $t$ test).

into $E P 4_{\text {Avil }}{ }^{-1}$ mice and WT littermates. Injection of SW033291 significantly increased the number of osteoblasts and decreased the number of adipocytes in WT mice, and these effects were absent in $E P 4_{A v i l}{ }^{-1}$ mice (Figure 4, G-I), indicating that the PGE2/EP4 sensory nerve axis regulates MSC differentiation. Moreover, MSCs isolated from $\mathrm{EP}_{\mathrm{Avil}}{ }^{-1-}$ mice showed an increase in CFU-AD and a decrease in CFU-OB and CFU-F populations compared with MSCs from WT littermates (Figure 4, J-M, and Supplemental Figure 3). Expression of the adipogenesis markers Pparg, Cebpa, and Fabp4 significantly increased, whereas expression of the osteogenesis markers Alp, Col1a1, and Runx2 significantly decreased in MSCs isolated from $E P 4_{\text {Avil }}{ }^{-1-}$ mice relative to WT mice in quantitative real-time PCR (qPCR) analysis (Supplemental Figure 4, D and E). The frequency of APCs $\left(\mathrm{CD} 45^{-} \mathrm{CD} 31^{-} \mathrm{Sca}-1^{+} \mathrm{CD} 24^{-}\right)$increased, whereas the frequencies of OPCs $\left(\mathrm{CD}^{-} 5^{-} \mathrm{CD} 31^{-} \mathrm{Sca}-1^{-} \mathrm{Pa}^{+}\right)$and MSCs $\left(\mathrm{CD} 45^{-} \mathrm{CD} 31^{-} \mathrm{Sca}-1^{+}\right.$ $\mathrm{CD} 24^{+}$) decreased (Figure $4, \mathrm{~N}-\mathrm{Q}$ ) in $\mathrm{EP}_{\text {Avil }}{ }^{-1-}$ mice relative to WT littermates. BrdU ${ }^{+} \mathrm{OPC}$ and $\mathrm{BrdU}^{+}$MSC levels decreased in
$E P 4_{A v i l}{ }^{-1-}$ mice compared with control mice, whereas $\mathrm{BrdU}^{+} \mathrm{APCs}$ did not significantly change in $E P 4_{A v i}{ }^{-1-}$ mice relative to WT littermates (Supplemental Figure 2, E-H). These results indicate that the EP4 receptor in sensory nerves is essential for sensory nerve regulation of MSCs differentiation.

PGE2 derived from osteoblasts regulates the differentiation of MSCs through sensory nerve EP4 by regulating sympathetic tone. We have shown that PGE2 derived from osteoblasts is primarily involved in sensory nerve regulation of bone formation (7). $\mathrm{COX} 2_{\mathrm{OCN}}{ }^{-1-}$ mice showed significant decreases in $\mathrm{BV} / \mathrm{TV}$ and $\mathrm{Tb}$.Th relative to WT littermates (Figure 5, A-C, and Supplemental Figure 5, A-C). Fat levels were significantly increased in the bone marrow of $\mathrm{COX} 2_{\text {OCN }}^{-1-}$ mice relative to WT controls, as evidenced by markedly higher frequencies of adipocytes and fat droplets in decalcified femurs stained with $\mathrm{OsO}_{4}$ (Figure 5, D-F). To examine whether PGE2 secreted from osteoblasts also regulates the differentiation of MSCs, we injected SW033291 into $\mathrm{COX} 2_{\text {OCN }}^{-1-}$ mice and WT littermates. We found that the effects 
A
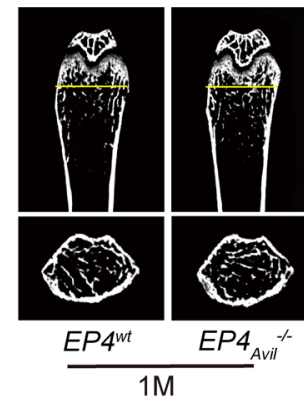

$\mathbf{E}$

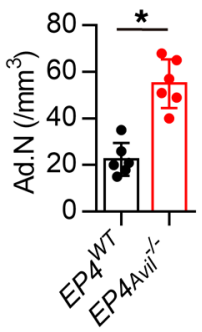

$\mathbf{F}$

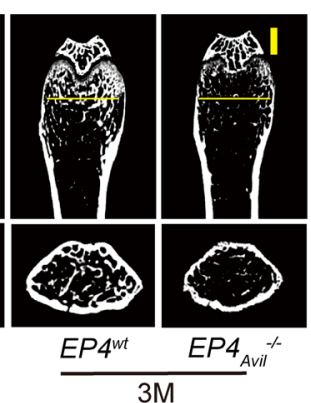

G

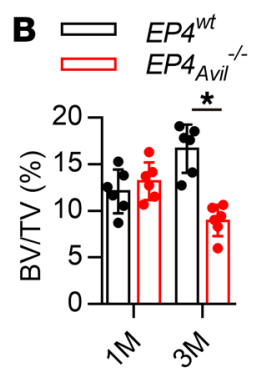

C

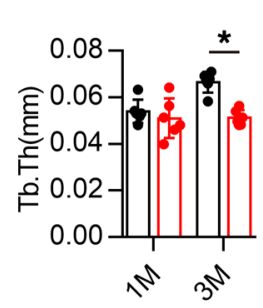

D

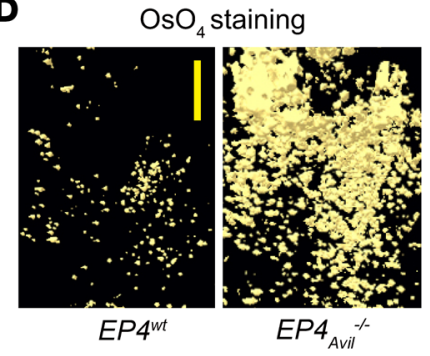

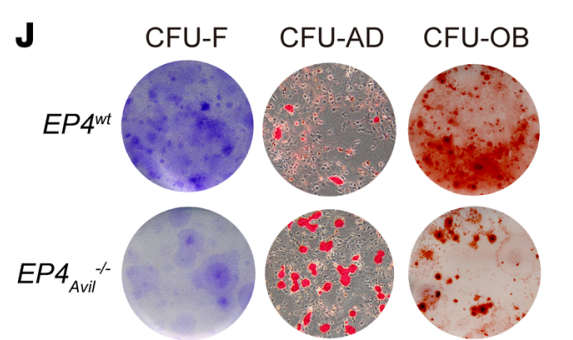

$\mathbf{K}$

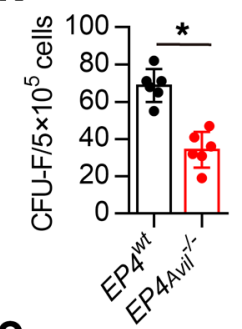

O

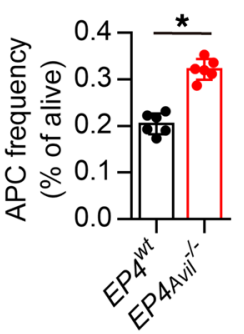

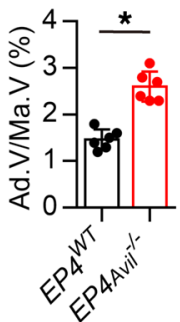

$\mathbf{L}$

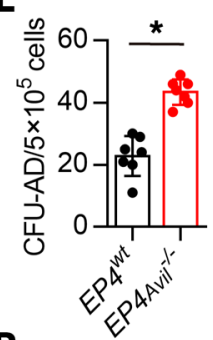

$\mathbf{P}$

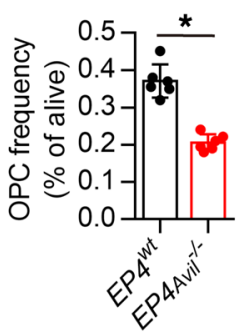

Perilipin
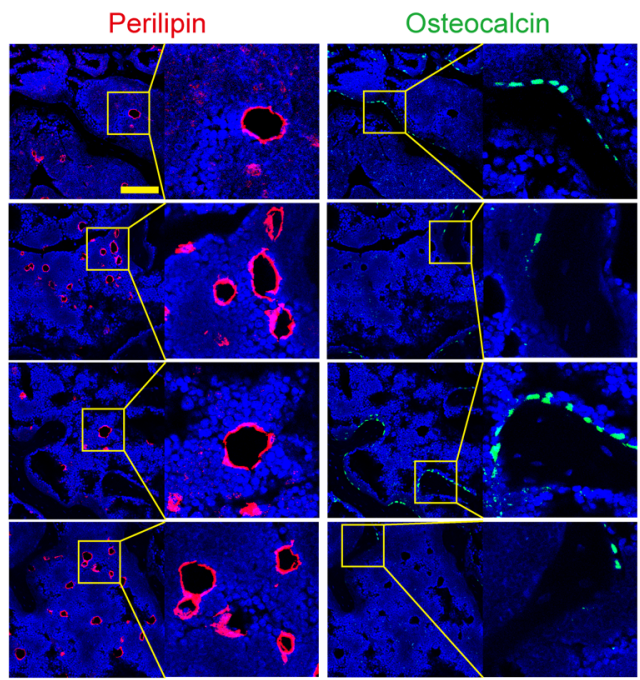

M

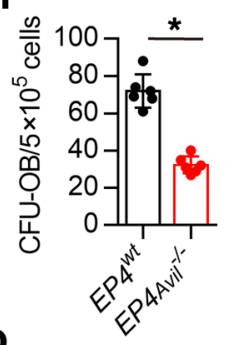

$\mathbf{Q}$

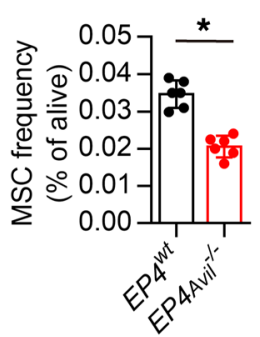

$\mathbf{N}$
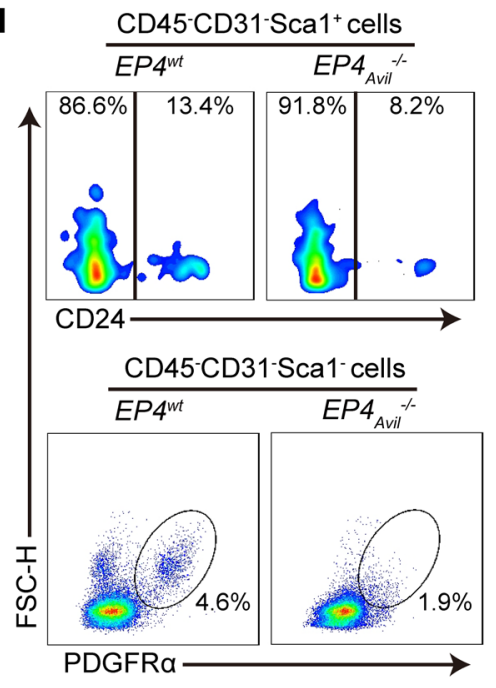

$\mathbf{H}_{\text {。 }}$

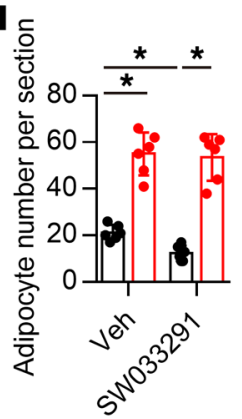

I

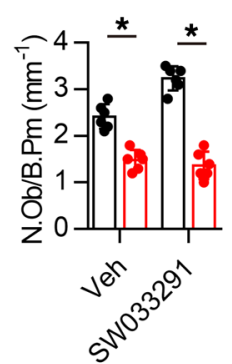

Figure 4. Deletion of the EP4 receptor in sensory nerves promotes adipogenesis and inhibits osteogenesis. (A-C) Representative $\mu C T$ images of femurs from 1- and 3-month-old male EP4 ${ }^{W T}$ and $E P 4_{\text {Avil }}{ }^{-1-}$ mice. The thin yellow lines indicate the area where the cross-section images were captured ( $0.5 \mathrm{~mm}$ proximal from the growth plate). Quantitative analysis of Tb.BV/TV and Tb.Th. Scale bar: $1 \mathrm{~mm}$. (D-F) Representative $\mu$ CT-detected $\mathrm{OsO}_{4}$-stained images of decalcified femurs and quantitative analysis of Ad.N and Ad.V/Ma.V in distal femurs from 3-month-old male EP4WT and $E P 4_{\text {Avil }}{ }^{-1-}$ mice. Scale bar: $500 \mu \mathrm{m}$. (G-I) Immunohistochemical staining of perilipin (red) and OCN (green) in femurs from 3-month-old male EP4WT and EP4 $4_{\text {Avil }}^{-/-}$mice treated with SW033291 (10 mg/kg/d) or vehicle for 1 month. Quantitative analysis of the density of perilipin and OCN in femurs from 3-month-old male EP4 ${ }^{W T}$ and EP4 ${ }_{\text {Avil }}^{-1-}$ mice treated with SW033291 or vehicle for 1 month. Scale bar: $50 \mu$ m. (J-M) Representative images of crystal violet-stained CFU-F, oil red O-stained CFU-AD, and alizarin red S-stained CFU-OB. Quantitative analysis of CFU-F, CFU-AD, and CFU-OB MSCs isolated from 3-month-old male EP4 ${ }^{W T}$ and EP4 ${ }_{\text {Avil }}{ }^{-/-}$mice. (N-Q) Representative dot plot images of flow cytometry and quantitative analysis of CD45-CD31-Sca-1+CD24- APCs, CD45-CD31-Sca-1-P $\alpha^{+}$OPCs, and CD45-CD31-Sca-1+CD24+ MSCs of live cells isolated from femurs of 3-month-old male $E P 4^{W T}$ and $E P 4_{\text {Avil }}{ }^{-/-}$mice. $n \geq 6$ per group; ${ }^{*} P<0.05$ (Student's $t$ test; 2 -way ANOVA for $\mathbf{H}$ and $\mathbf{I}$ ). 
A

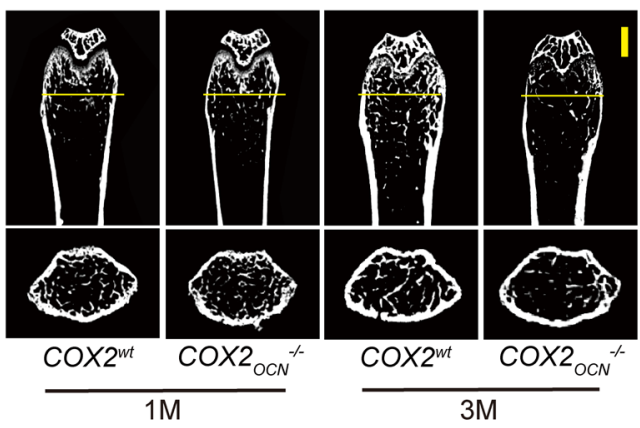

B

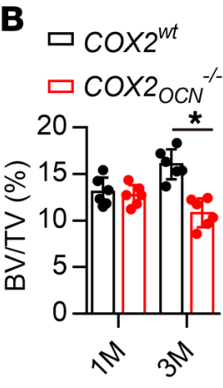

C

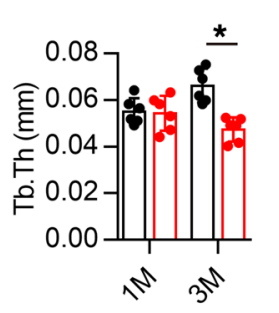

D

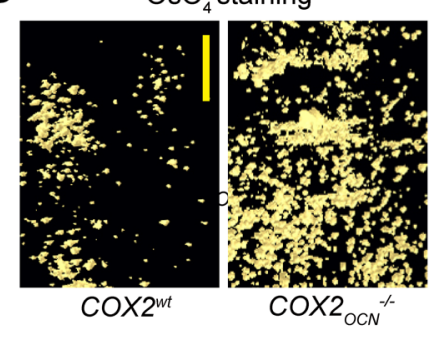

E

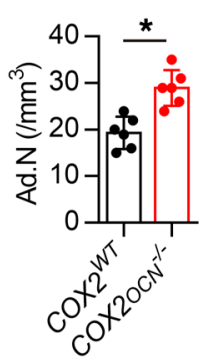

$\mathbf{F}$

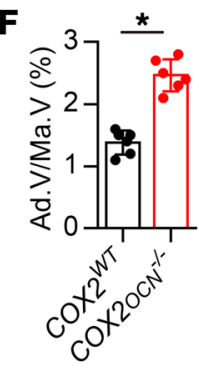

J

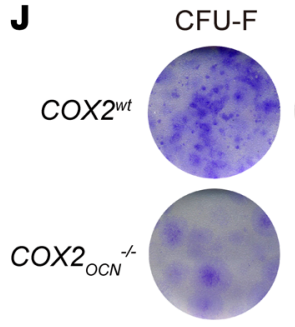

$\mathbf{K}$
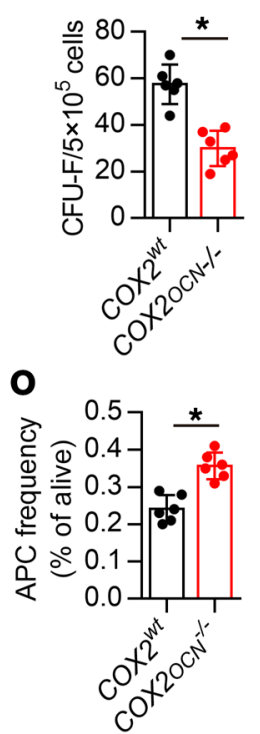

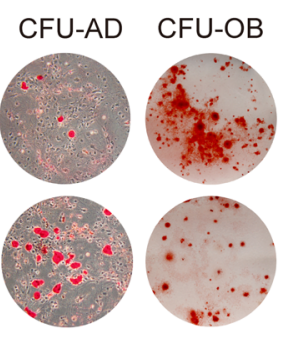

$\mathbf{L}$
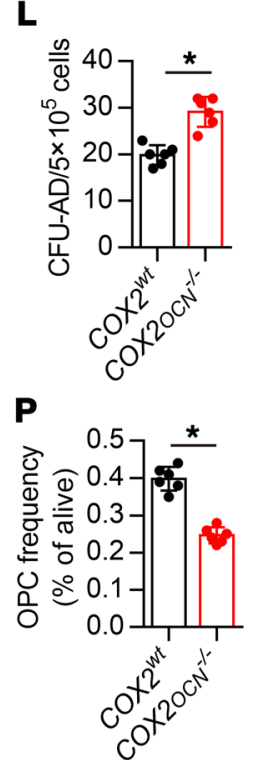

G
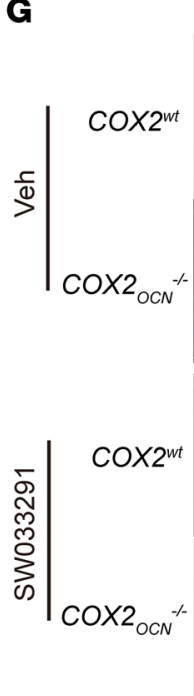

M
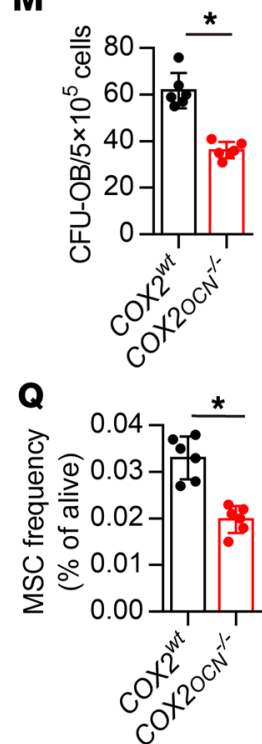

Perilipin

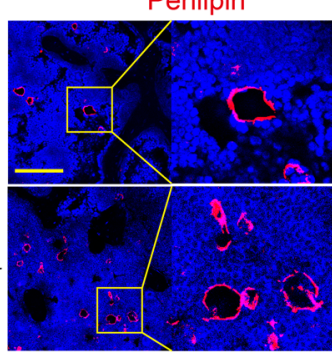

Osteocalcin
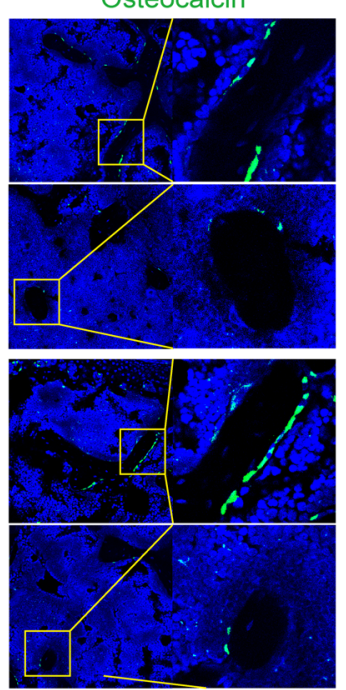

$\mathbf{N}$

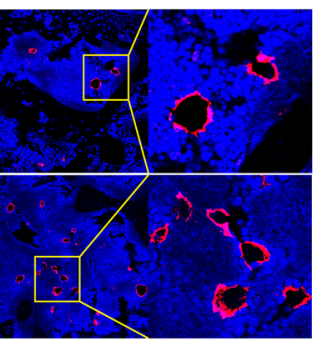

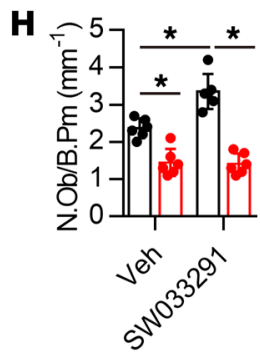

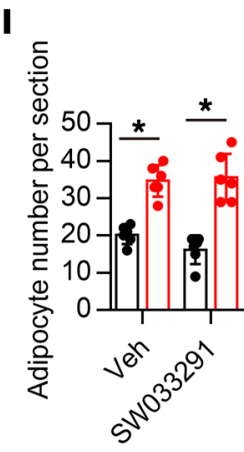

Figure 5. PGE2 derived from osteoblasts regulates the differentiation of MSCs through EP4 in sensory nerves. (A-C) Representative $\mu$ CT images of femurs from 1- and 3-month-old male COX2 ${ }^{W T}$ and $C O X 2_{\text {ocN }}^{-1-}$ mice. The thin yellow lines indicate the area where the cross-section images were captured ( $0.5 \mathrm{~mm}$ proximal from the growth plate). Quantitative analysis of Tb.BV/TV and Tb.Th. Scale bar: $1 \mathrm{~mm}$. (D-F) Representative $\mu$ CT-detected OsO -stained

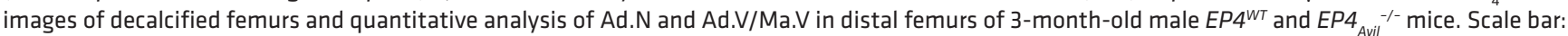
$500 \mu \mathrm{m}$. (G-I) Representative images of immunohistochemical staining of perilipin (red) and OCN (green) in femurs of 3-month-old male COX2 ${ }^{W T}$ and COX $2_{\text {OCN }}{ }^{-/-}$mice treated with SW033291 (10 mg/kg/d) or vehicle for 1 month. Quantitative analysis of density of perilipin and OCN in femurs of 3-month-old male COX2 ${ }^{W T}$ and $C O X 2_{O C N}^{-1-}$ mice treated with SW033291 or vehicle for 1 month. Scale bar: $50 \mu \mathrm{m}$. (J-M) Representative images of crystal violet-stained CFU-F, oil red O-stained CFU-AD, and alizarin red S-stained CFU-OB. Quantitative analysis of CFU-F, CFU-AD, and CFU-OB MSCs isolated from 3-monthold male COX2 ${ }^{W T}$ and $C O X 2_{\text {OCN }}^{-1-}$ mice. (N-Q) Representative dot plots of flow cytometry and quantitative analysis of CD45-CD31-Sca-1+CD24- APCs, CD45 CD31-Sca-1-PDGFR $\alpha^{+}\left(\mathrm{P}^{+}\right)$OPCs, and CD45-CD31-Sca-1+CD24+ MSCs of live cells isolated from femurs of 3-month-old male COX2 ${ }^{W T}$ and COX2 ${ }^{-1-}$ mice. $n \geq$ 6 per group; ${ }^{*} P<0.05$ (Student's $t$ test for B, C, E, F, K-M, and $\mathbf{O}-\mathbf{Q}$; 2-way ANOVA for $\mathbf{H}$ and I). 
A

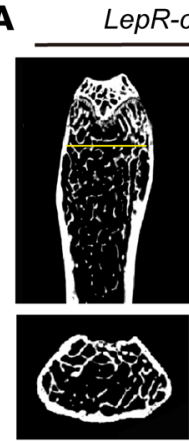

Veh
B

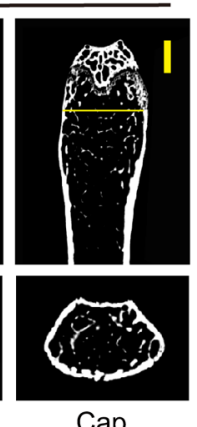

Cap

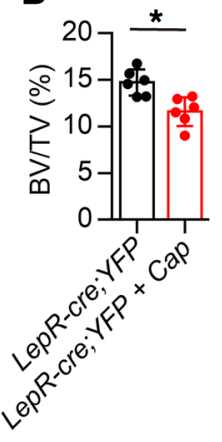

Perilipin

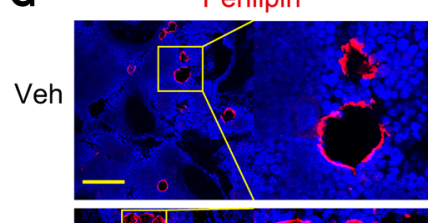

G

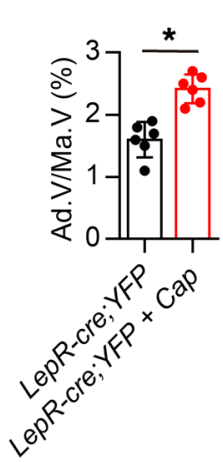

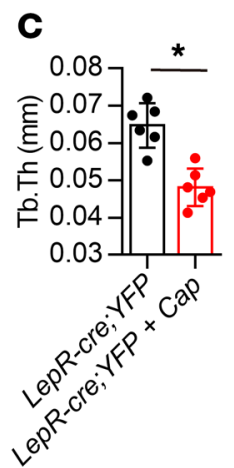

D

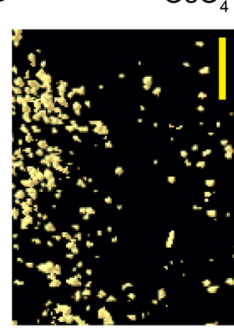

Veh
$\mathrm{OsO}_{4}$ staining

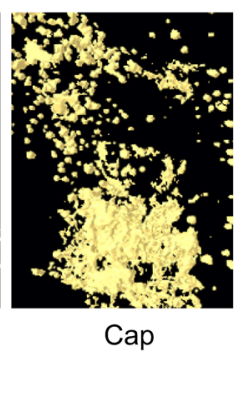

E

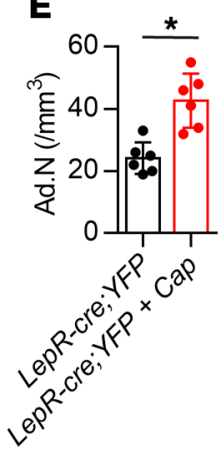

I

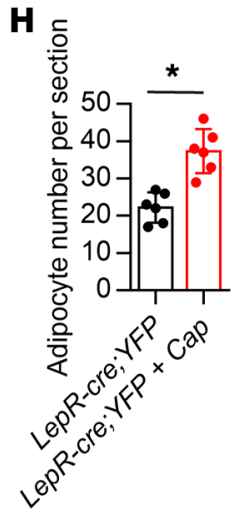

Osteocalcin

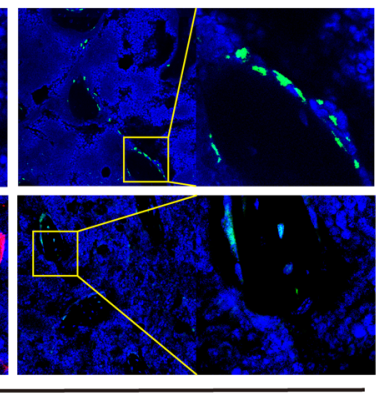

LepR-cre;YFP

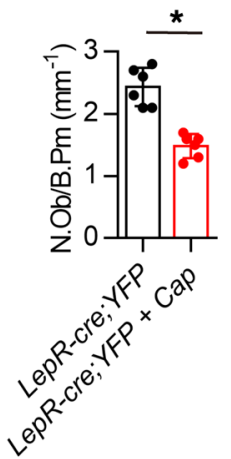

Figure 6. Capsaicin-induced sensory nerve denervation promotes adipogenesis and inhibits osteogenesis. (A-C) Representative $\mu C T$ images and quantitative analysis of Tb. BV/TV and Tb.Th of femurs from 3-month-old male LepR-Cre;YFP mice injected with capsaicin (Cap; $30 \mathrm{mg} / \mathrm{kg} / \mathrm{d}$ ) or vehicle for 1 week and euthanized after another 2 weeks. The yellow lines indicated the area where the cross-section images were captured ( $0.5 \mathrm{~mm}$ proximal from the growth plate). Scale bar: $1 \mathrm{~mm}$. (D-F) Representative $\mu \mathrm{CT}$-detected $\mathrm{OsO}_{4}$-stained images of decalcified femurs and quantitative analysis of Ad.N and Ad.V/ Ma.V in distal femurs of 3-month-old male LepR-Cre; YFP mice injected with capsaicin or vehicle for 1 week and euthanized after another 2 weeks. Scale bar: $500 \mu \mathrm{m}$. (G-I) Representative images of immunohistochemical staining of perilipin (red) and OCN (green) in femurs of 3-month-old male LepR-Cre; YFP mice injected with capsaicin or vehicle for 1 week and euthanized after another 2 weeks. Scale bar: $50 \mu \mathrm{m}$. $n \geq 6$ per group; ${ }^{*} P<0.05$ (Student's $t$ test).

of SW033291 on osteogenesis induction and adipogenesis inhibition were reduced in $\mathrm{COX} 2_{\mathrm{OCN}^{-/}}^{-1}$ mice (Figure 5, G-I), indicating that PGE2 derived from osteoblasts in the bone remodeling microenvironment is essential in the regulation of MSC differentiation. These results were confirmed in CFU-F, CFU-OB, and CFU-AD assays (Figure 5, J-M, and Supplemental Figure 3) and qPCR analysis of osteogenic and adipogenic markers (Supplemental Figure 5, D and E). Moreover, flow cytometry analysis also demonstrated decreased frequency of OPCs and MSCs, and increased frequency of APCs in $\mathrm{COX} 2_{\mathrm{OCN}}^{-1-}$ mice (Figure 5, $\mathrm{N}-\mathrm{Q}$ ). Frequencies of BrdU-incorporated OPCs and MSCs were decreased, whereas BrdU-incorporated APCs and total cell viability were not affected (Supplemental Figure 2, I-N). These results indicate that PGE2 derived from osteoblasts in the bone remodeling microenvironment act on sensory nerves for differentiation of MSCs.

We have shown that PGE2 activation of sensory nerve regulates bone formation by tuning down sympathetic tone (7). We also found that obstruction of the PGE2/EP4 sensory nerve axis induced an increase in sympathetic activity (Supplemental Figure 6). In this case, to further test whether the sympathetic nerve also regulates MSC differentiation, we injected propranolol into $E P 4_{A v i l}{ }^{-/}$mice and WT littermates. Propranolol rescued bone loss in $E P 4_{A v i l}^{-1-}$ mice (Supplemental Figure 7, A-C). Importantly, propranolol promoted osteogenesis and inhibited adipogenesis in $E P 4_{A v i}{ }^{-1-}$ mice (Supplemental Figure 7, D-F). To further examine the effect of specific sympathetic adrenoceptors in sensory nerve-regulated MSC differentiation, we injected a selective $\beta_{1}$ adrenoceptor antagonist (atenolol), $\beta_{2}$ adrenoceptor antagonist (ICI118551), and $\beta_{3}$ adrenoceptor antagonist (SR59230A) into $E P 4_{A v i}{ }^{-1-}$ mice and WT littermates. The results showed that only $\beta_{2}$ adrenoceptor antagonist injection had an effect similar to that of propranolol injection, rescuing the bone loss and enhanced adipogenesis phenotype of $E P 4_{\text {Avil }}{ }^{-1}$ mice (Supplemental Figure 8). The sympathetic $\beta_{2}$ adrenoceptor antagonist's attenuation of sensory nerve regulation of bone formation suggests regulation of MSCs by sympathetic tone.

$L e p R^{+} M S C s$ are the major source of the increase in adipocytes, and are regulated by the PGE2/EP4 sensory nerve axis. In the experiments described above, the results showed that the frequencies of MSCs and OPCs decreased, whereas the frequency of APCs increased. However, the specific in vivo population of MSCs that responded to sensory nerve regulation and the origin of these increased adipocytes in sensory nerve-denervated mice remained elusive. To address this question, we used the leptin receptor-Cre (LepR-Cre) mouse line to fate map MSCs in vivo according to 
A

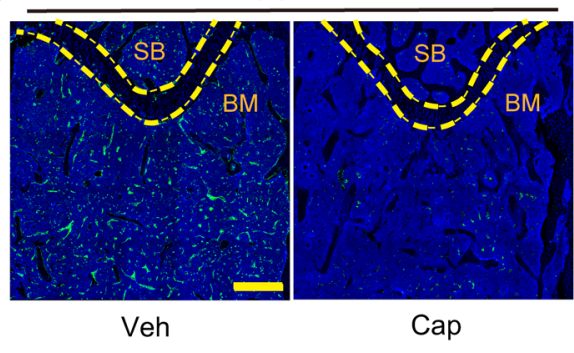

LepR-cre;YFP
B

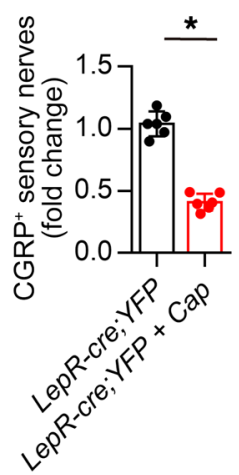

C

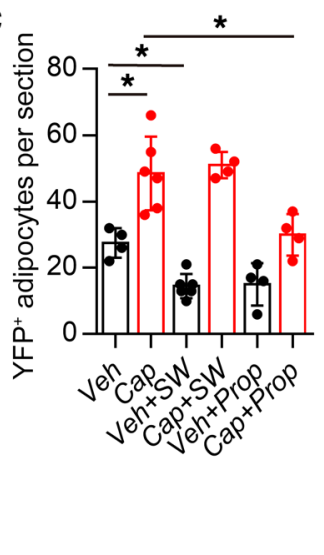

D

Perilipin/YFP
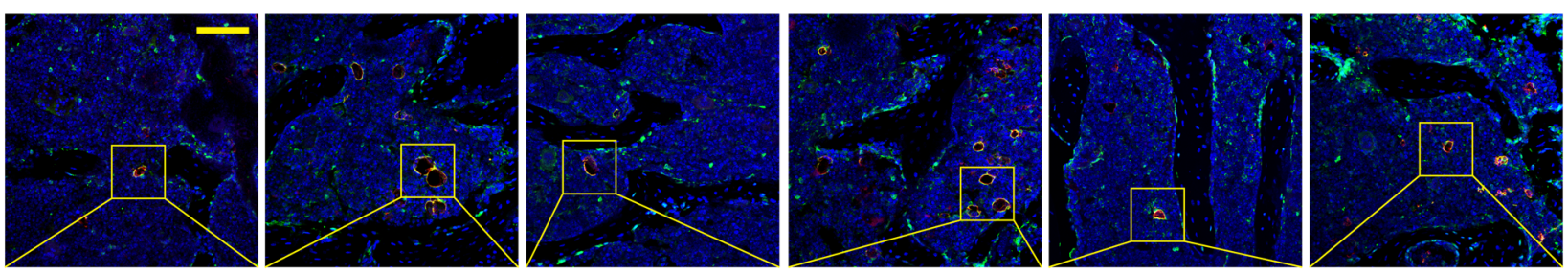

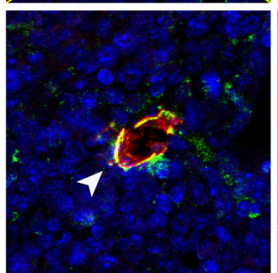

Veh

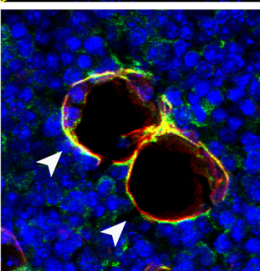

Cap

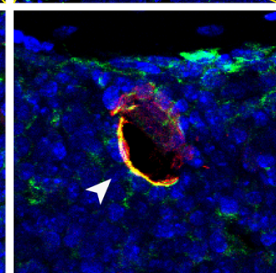

Veh+SW

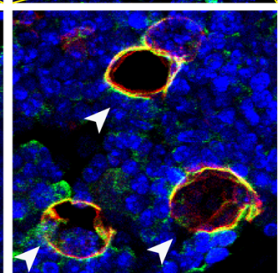

$\mathrm{Cap}+\mathrm{SW}$

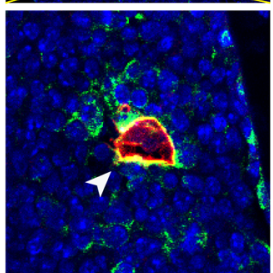

Veh+Prop

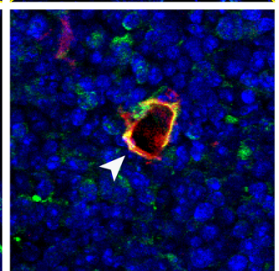

Cap+Prop

LepR-cre;YFP

Figure 7. LepR ${ }^{+}$MSCs are the major source of the increase in adipocytes and are regulated by the PGE2/EP4 sensory nerve axis. (A and B) Representative images of immunofluorescence staining and quantitative analysis of CGRP+ sensory nerves (green) in distal femurs of 3-month-old male LepR-Cre; YFP mice injected with capsaicin or vehicle for 1 week and euthanized after another 2 weeks (SB, subchondral bone). Scale bar: $100 \mu \mathrm{m}$. (C) Representative images of immunofluorescence staining of colocalization of perilipin (red) and YFP (representing LepR+ cells) (green) in femur bone marrow from 3-month-old LepR-Cre;YFP mice treated with capsaicin (30 mg/kg/d for 1 week), SW033291 (10 mg/kg/d for $1 \mathrm{month}$ ), or propranolol (0.5 mg/kg/d for 6 weeks). (D) Quantitative analysis of YFP+ adipocytes for each of the groups (marrow adipocytes labeled by white arrowheads). Scale bar: $50 \mu \mathrm{m}$. $n \geq 6$ per group; ${ }^{*} P<0.05$ (Student's $t$ test for $\mathbf{B}, 2$-way ANOVA for $\mathbf{C}$ ).

previous reports (33); we crossed LepR-Cre with Rosa26-YFP mice to generate $L e p R$-Cre;YFP mice for an in vivo MSC fate mapping assay. Capsaicin or vehicle was injected into 3-month-old LepR-Cre;YFP mice for 1 week to generate the induced sensory denervation model, and mice were euthanized after another 2 weeks. The sensory denervation efficacy of capsaicin injection was comparable to that in $T r k A_{A v i l}{ }^{-1-}$ mice, as verified by von Frey tests, hot plate tests, and TrkA immunostaining assay (Supplemental Figure 9). Micro-CT ( $\mu \mathrm{CT}$ ) analysis showed that BV/TV and Tb.Th were significantly reduced in the capsaicin compared with the vehicle injection group (Figure 6, A-C). Fat levels were significantly increased in the bone marrow of capsaicin-injected mice relative to vehicle-injected controls, as evidenced by markedly higher levels of adipocytes and fat droplets in decalcified femurs stained with $\mathrm{OsO}_{4}$ (Figure 6, D-F). Adipogenesis was increased and osteogenesis was decreased after capsaicin relative to control injection (Figure 6, G-I). Immunostaining showed a significant decrease in calcitonin gene-related peptide-positive (CGRP ${ }^{+}$) sensory nerves in bone marrow, which validated the sensory denervation efficacy of capsaicin injection (Figure 7, A and B). In vivo fate mapping assays showed that $\mathrm{YFP}^{+}$adipocytes were significantly increased after sensory denervation. Propranolol, but not SW033291, lowered the number of $\mathrm{YFP}^{+}$adipocytes that were induced by sensory denervation (Figure 7, C and D). These data together indicate that $\mathrm{LepR}^{+}$MSCs respond to sensory nerve denervation and commit to adipogenic differentiation while yielding osteogenesis of MSCs.

Impairment of EP4 in sensory nerves promotes adipogenesis and attenuates bone regeneration. To examine whether sensory nerves also regulate bone regeneration, we created a bone marrow ablation/bone regeneration model in LepR-Cre;YFP mice with impairment of sensory nerves by injection with capsaicin or vehicle. Bone regeneration was significantly reduced with sensory denervation by injection of capsaicin in $\mu \mathrm{CT}$ analysis, SO/ FG staining (red, cartilage; green, bone), Masson's staining (red, 
A

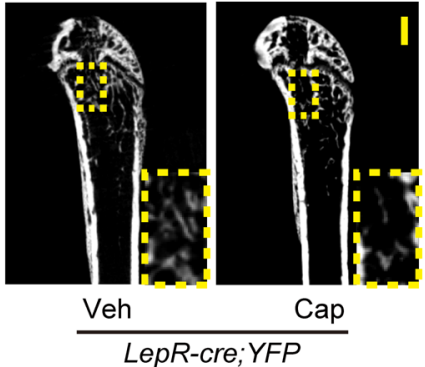

D
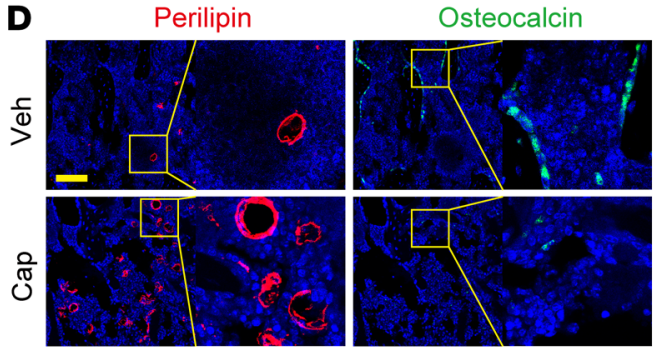

LepR-cre;YFP
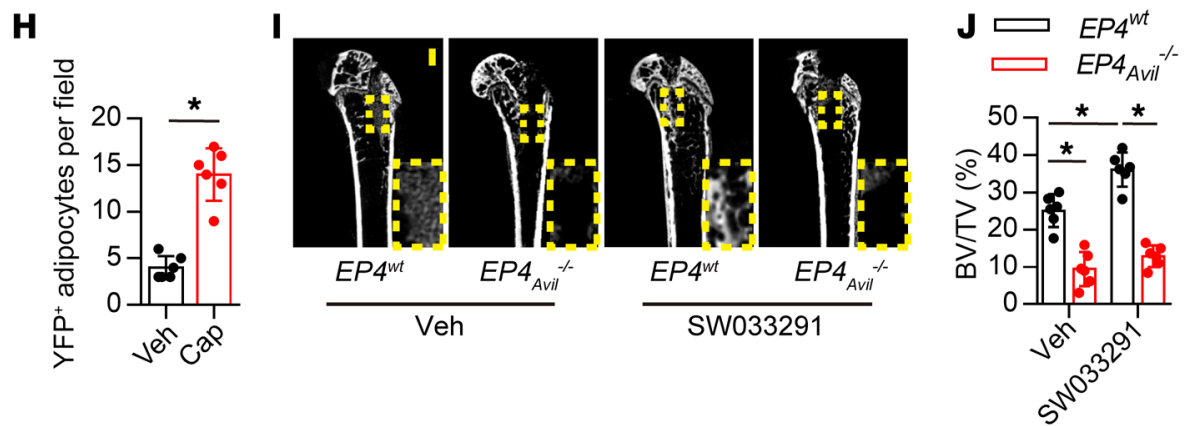

E

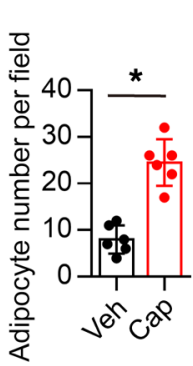

$\mathbf{F}$
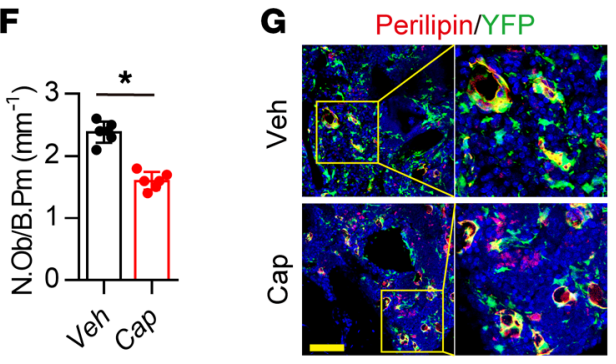

LepR-cre;YFP

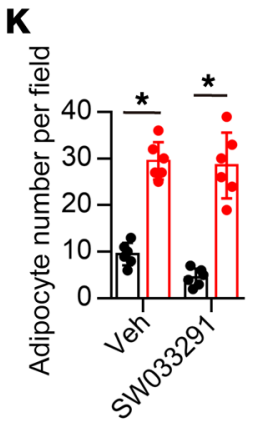

$\mathbf{L}$
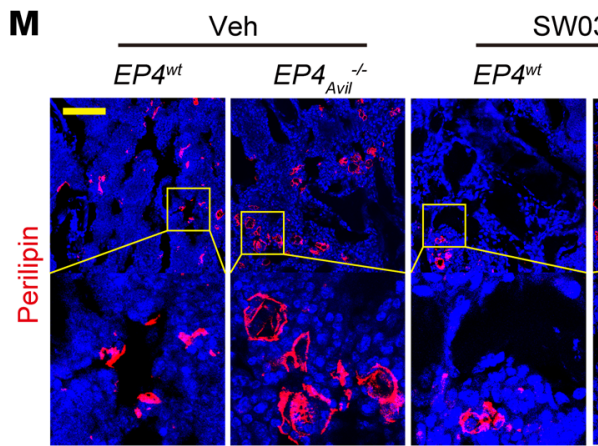

SW033291
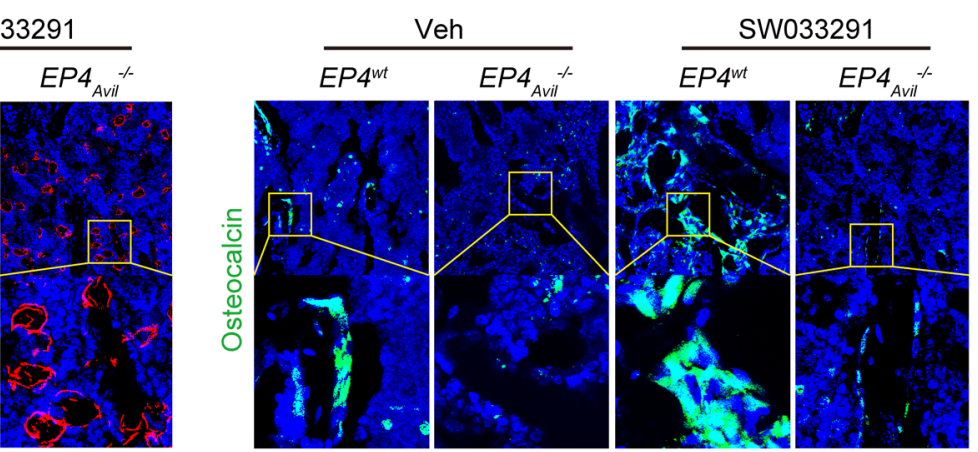

Figure 8. Impairment of EP4 sensory nerves promotes adipogenesis and attenuates bone regeneration. (A and B) Representative $\mu$ CT images of bone regeneration 7 days after femoral bone marrow ablation in 3-month-old male LepR-Cre;YFP mice treated with capsaicin (30 mg/kg/d) or vehicle. Scale bar: $1 \mathrm{~mm}$. Areas selected for measurement of BV/TV are indicated by yellow squares. (C) Representative SO/FG and Masson's staining (red, muscle and cytoplasm; blue, bone) images in the regeneration area in 3-month-old male LepR-Cre; YFP mice treated with capsaicin or vehicle 7 days after bone marrow ablation. Red represents cartilage area; green represents woven bone area in SO/FG staining. Scale bars: $100 \mu \mathrm{m}$. (D-F) Representative images and analysis of perilipin (red) and OCN (green) staining in the regeneration area from the capsaicin-treated and control groups. Scale bar: $50 \mu \mathrm{m}$. (G and $\mathbf{H}$ ) Representative images of immunofluorescence staining of colocalization of perilipin (red) and YFP (representing LepR ${ }^{+}$ cells; green), and quantitative analysis of density of $\mathrm{YFP}^{+}$adipocytes in the regeneration area from capsaicin-treated and control groups. Scale bar: $100 \mu \mathrm{m}$. (I and J) Representative $\mu \mathrm{CT}$ images of bone regeneration after femoral bone marrow ablation in 3-month-old male $E P 4^{W T}$ and $E P 4_{\text {Avil }}{ }^{-1-}$ mice treated with $10 \mathrm{mg} / \mathrm{kg} / \mathrm{d}$ SW033291 or vehicle 7 days after bone marrow ablation. Scale bar: $1 \mathrm{~mm}$. Selected areas for the measurements of BV/TV are indicated by yellow squares. (K-M) Immunohistochemical staining and quantitative analysis of perilipin (red) and OCN (green) in the regeneration area from 3-month-old male $E P 4^{W T}$ and $E P 4_{\text {Avil }}{ }^{-1-}$ mice treated with $10 \mathrm{mg} / \mathrm{kg} / \mathrm{d}$ SW033291 or vehicle. Scale bar: $50 \mu \mathrm{m}$. $n \geq 6$ per group; ${ }^{*} P<0.05$ (Student's $t$ test for $\mathbf{B}, \mathbf{E}, \mathbf{F}$, and $\mathbf{H}$; 2-way ANOVA for $\mathbf{J}-\mathbf{L}$ ). 
A

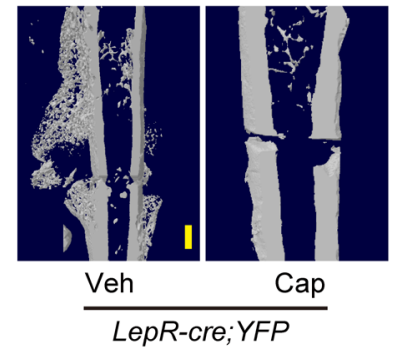

B

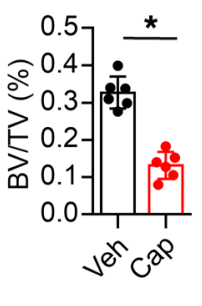

C

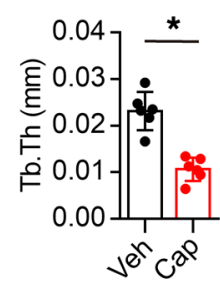

D

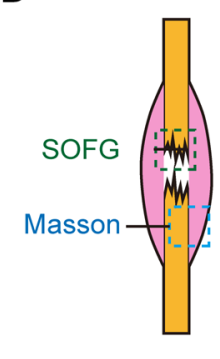

LepR-cre;YFP

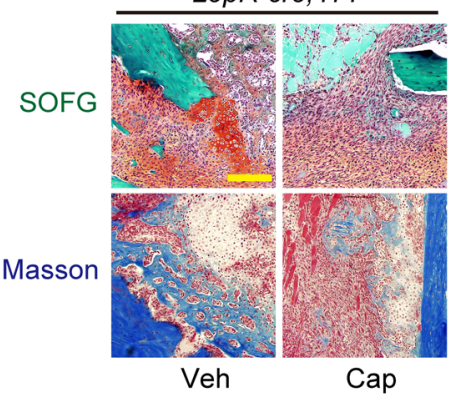

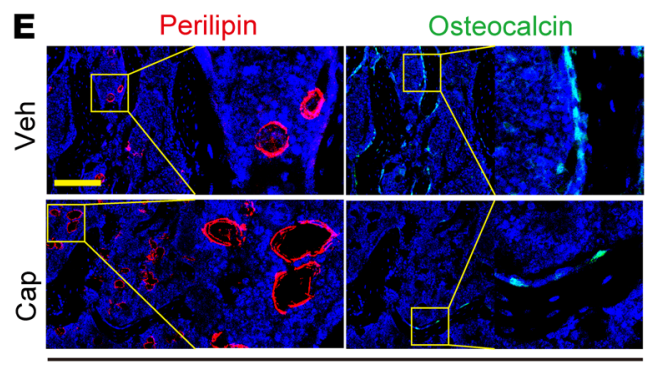

LepR-cre;YFP
$\mathbf{F}$

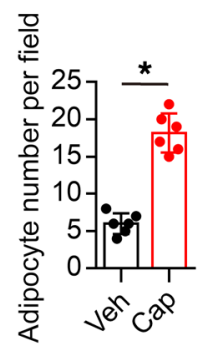

G

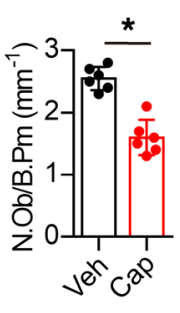

H

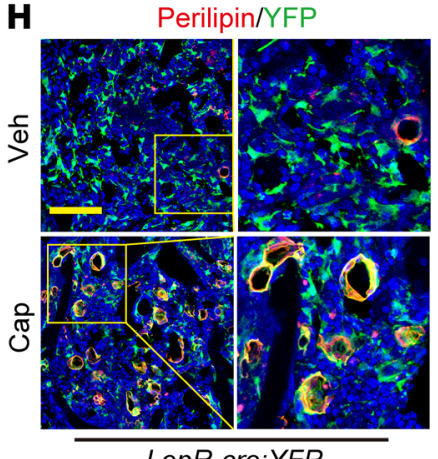

I

LepR-cre;YFP

Figure 9. Sensory nerve denervation impaired bone fracture healing. (A-C) Representative $\mu$ CT images and quantitative analysis of BV/TV and Tb.Th of fracture healing area in 3-month-old male LepR-Cre;YFP mice treated with capsaicin (30 mg/kg/d) or vehicle 2 weeks after bone fracture. Scale bar: $1 \mathrm{~mm}$. (D) Representative SO/FG (red, cartilage; green, bone) and Masson's staining (red, muscle and cytoplasm; blue, bone) images in the fracture healing area in the capsaicin-treated and control groups. Scale bar: $100 \mu \mathrm{m}$. (E-C) Representative images of immunohistochemical staining and quantitative analysis of density of perilipin (red) and OCN (green) in the fracture healing area in the capsaicin-treated and control groups. Scale bar: $50 \mu \mathrm{m}$. (H and I) Representative images of immunofluorescence staining of colocalization of perilipin (red) and YFP (representing LepR ${ }^{+}$cells; green), and quantitative analysis of the density of YFP+ adipocytes in fracture healing area in the capsaicin-treated and control groups. Scale bar: $50 \mu \mathrm{m} . n \geq 5$ per group; ${ }^{*} P<0.05$ (Student's $t$ test).

muscle and cytoplasm; blue, bone), and Movat pentachrome staining (yellow, bone; green, cartilage/endochondral ossification; red, bone marrow) (Figure 8, A-C, and Supplemental Figure 10A). Costaining of perilipin with OCN showed active osteoblast differentiation, with few perilipin-positive adipocytes during bone regeneration in the vehicle group. In contrast, while $\mathrm{OCN}^{+}$ osteoblast levels were reduced, adipocyte numbers increased significantly with injection of capsaicin (Figure 8, D-F). Again, fate mapping assays showed that the adipocytes accounting for the increase were largely $\mathrm{YFP}^{+}$(Figure 8, G and $\mathrm{H}$ ). We further tested whether EP4 in sensory nerves mediates bone regeneration by deleting EP4 in sensory nerves of $E P 4_{\text {Avil }}{ }^{-1}$ mice. Bone regeneration decreased significantly in $E P 4_{A v i l}{ }^{-1-}$ mice relative to WT littermates (Figure 8, I and J). SW033291 stimulated bone regeneration, and this effect was attenuated in $E P 4_{A v i l}{ }^{-1}$ mice (Figure 8, I and J). Moreover, the number of osteoblasts increased and the number of adipocytes decreased in the bone-regenerative region of WT mice treated with SW033291. In contrast, the number of adipocytes increased and the number of osteoblasts decreased in the bone-regenerative region of $\mathrm{EP}_{\text {Avil }}{ }^{-1}$ mice relative to WT littermates (Figure 8, K-M). We also tested whether the EP4 receptor in LepR ${ }^{+}$MSCs was involved in sensory nerve regulation of bone formation. We crossed LepR-Cre mice with $E P 4^{W T}$ mice to generate $E P 4_{L e p R}{ }^{-1}$ descendants. We observed no significant changes in bone parameters in either 1- or 3-month- old $E P 4_{\text {LepR }}^{-/-}$mice relative to WT littermates (Supplemental Figure 11, A-C). There were no significant differences in marrow fat, adipogenesis, or osteogenesis between $E P 4_{\text {LepR }}^{-1 /}$ mice and WT littermates, as shown by $\mu \mathrm{CT}$ analysis of decalcified femurs stained with $\mathrm{OsO}_{4}\left(\right.$ Supplemental Figure 11, D-F). Both EP4 $4_{\text {LepR }}{ }^{-1-}$ mice and WT littermates showed decreased adipogenesis and increased osteogenesis with injection of SW033291 (Supplemental Figure 11, G-I). In addition, we eliminated EP4 expression in osteoblasts by crossing $O C N$-Cre mice with $E P 4^{W T}$ mice to generate $E P 4_{O C N}{ }^{-1-}$ descendants; however, like $E P 4_{L e p R}{ }^{-1-}$ mice, $E P 4_{O C N}{ }^{-1-}$ mice showed no bone or MSC alteration relative to WT littermates (Supplemental Figure 12, A-F). These results indicate that EP4 in sensory nerves, but not in MSCs or osteoblasts, regulates differentiation of MSCs during bone regeneration.

Impairment of EP4 in sensory nerves interrupts bone fracture healing. To examine whether EP4 in sensory nerves regulates bone fracture healing, we created a bone fracture model in LepR-Cre;YFP mice with sensory nerve denervation by injecting capsaicin or vehicle. Injection of capsaicin reduced BV relative to vehicle treatment in $\mu \mathrm{CT}$ analysis (Figure 9, A-C), and bone formation was markedly lower in the capsaicin group, as shown by SO/FG staining (red, cartilage; green, bone) (Figure 9D). Masson's staining (red, muscle and cytoplasm; blue, bone) and Movat pentachrome staining (yellow, bone; green, cartilage/endochondral ossification; red, bone marrow) showed that 
A

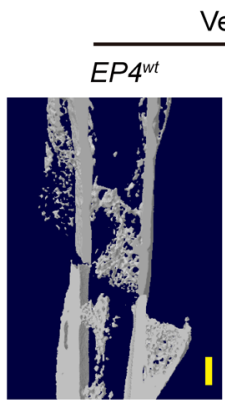

D

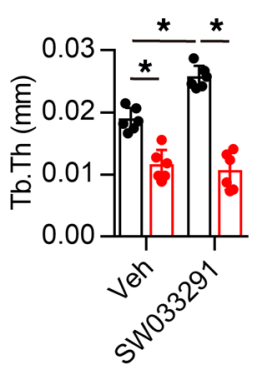

$\mathbf{E}$

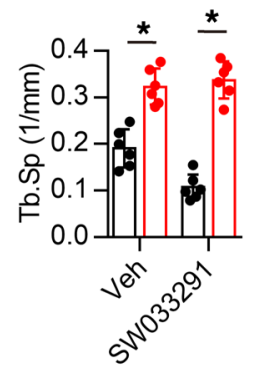

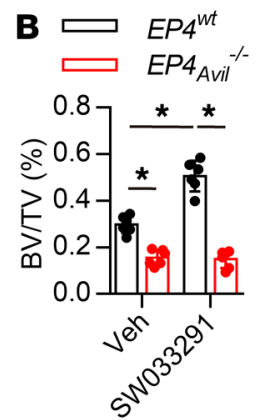

C

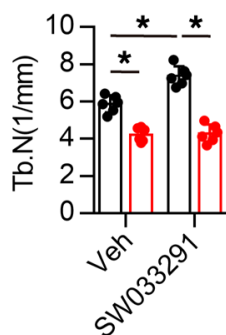

F

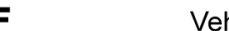

G

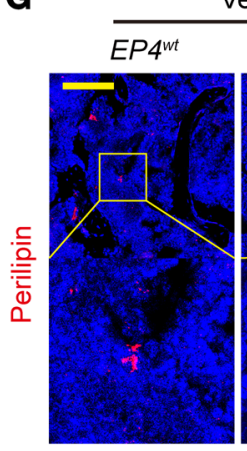

Veh $E P 4_{\text {Avil }}{ }^{-1}$

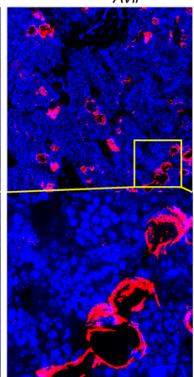

H

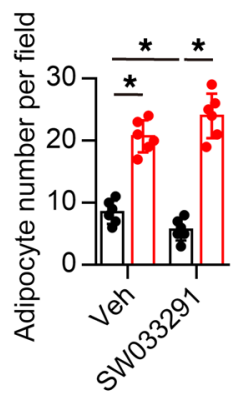

I

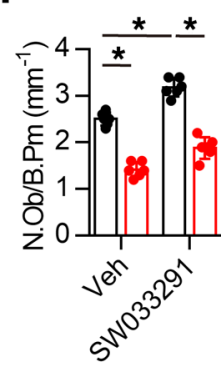

SW033291

$$
\text { EP4 }{ }^{w t}
$$
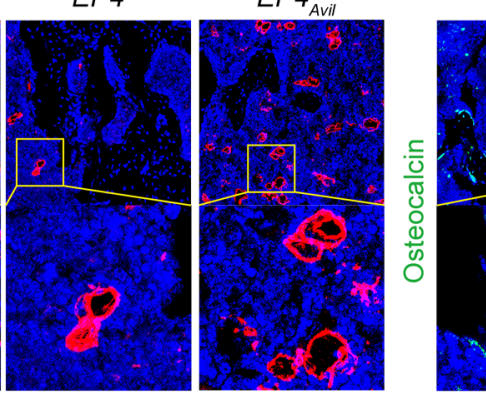

Figure 10. Impairment of EP4 sensory nerves interrupts bone fracture healing. (A-E) Representative $\mu \mathrm{CT}$ images and quantitative analysis of BV/TV, Tb.Th, Tb.N, and Tb.Sp of bone fracture healing area in 3-monthold male $E P 4^{W T}$ and $E P 4_{\text {Avil }}{ }^{-1-}$ mice treated with $10 \mathrm{mg} / \mathrm{kg} / \mathrm{d}$ SW033291 or vehicle 2 weeks after bone fracture. Scale bar: $2 \mathrm{~mm}$. (F) Representative images of SO/FG and Masson's staining and (G-I) immunohistochemical staining and quantitative analysis of perilipin (red) and OCN (green) in the fracture healing area in 3-month-old male EP4 ${ }^{W T}$ and $E P 4_{A v i l}{ }^{-1-}$ mice treated with SW033291 or vehicle 2 weeks after bone fracture. Scale bar: $50 \mu \mathrm{m}$. $n \geq 5$ per group; ${ }^{*} P<0.05$ (2-way ANOVA).

bone callus volume was also dramatically decreased with injection of capsaicin (Figure 9D and Supplemental Figure 10B). As expected, costaining of perilipin with $\mathrm{OCN}$ showed fewer $\mathrm{OCN}^{+}$ osteoblasts, whereas adipocyte numbers increased significantly with injection of capsaicin (Figure 9, E-G). Importantly, the adipocytes accounting for the increase were primarily $\mathrm{YFP}^{+}$, indicating they were descendants of LepR ${ }^{+}$cells (Figure 9, H and I). We then performed bone fracture surgery on $\mathrm{EP} 4_{\text {Avil }}^{-/-}$mice and WT littermates to examine whether EP4 signaling mediates sensory nerve regulation. BV/TV and formation were significantly reduced in the bone healing region of $\mathrm{EP}_{\mathrm{Avil}}{ }^{-/-}$mice relative to
WT littermates (Figure 10, A-E). Injection of SW033291 stimulated bone formation in the bone healing region of WT mice, but such effects were absent in EP4 ${ }_{\text {Avil }}{ }^{-1-}$ mice (Figure 10, A-E). Moreover, the SW033291-induced increase in bone callus formation observed in WT mice was absent in EP4 $4_{\text {Avil }}^{-1-}$ mice, as shown by SO/FG, Masson's, and Movat pentachrome staining (Figure 10F and Supplemental Figure 10C). To determine whether sensory nerves stimulated the healing of bone fracture by regulation of MSC differentiation, we coimmunostained sections of the fracture region with perilipin and OCN. The number of adipocytes increased significantly in $E P 4_{A v i l}{ }^{-/-}$mice relative to 
WT littermates, with or without injection of SW033291, whereas SW033291 significantly increased the number of $\mathrm{OCN}^{+}$osteoblasts in the bone healing region of WT mice, but such effects were absent in $\mathrm{EP}_{\text {Avil }}{ }^{-/-}$mice (Figure 10, G-I). Taken together, these results indicate that sensory nerves regulate osteoblast differentiation of MSCs for bone fracture healing through PGE2/ EP4 signaling.

\section{Discussion}

Bone marrow MSCs differentiate into osteoblasts, adipocytes, and chondrocytes to maintain bone integrity (22-24). The maintenance of MSCs and their potential commitment to different cell lineages is essential for bone homeostasis $(15,21,34)$. Notably, recent studies also characterized skeletogenic cells - which could differentiate to downstream progenitors of bone, cartilage, and stromal tissue - as skeletal stem cells (35-40). The MSCs committed as progenitors, including OPCs and APCs, could further differentiate into mature osteoblasts and adipocytes $(33,41)$. Plentiful markers have been developed to identify MSCs or their descendants for accurate analysis (42-46). A previous study showed that CD $45^{-}$CD $31^{-}$Sca- $1^{+}$CD $24^{+}$MSCs occupied more than $90 \%$ of the colonies formed with a trilineage differentiation capability assay (33). In the present study, we used CD $45^{-} \mathrm{CD} 31^{-} \mathrm{Sca}-1^{+} \mathrm{CD} 24^{+}$to

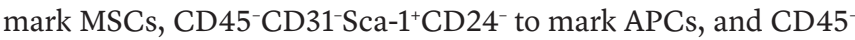
CD31-Sca-1-PDGFR $\alpha^{+}\left(\mathrm{P}^{+}\right)$to mark OPCs. We found that sensory nerves regulate the fate of MSCs by tuning sympathetic nerve tones through binding of the bone-forming signal PGE2 to the EP4 receptor; this result provides the first direct evidence to our knowledge of sensory nerves as a niche for mammalian stem cells.

The frequency of MSCs was reduced and their ability to differentiate into CFU-Fs impeded in sensory denervation mice, which increased the potential for adipogenesis and impaired osteogenesis both in vivo and in vitro (Supplemental Figure 3 and Supplemental Figure 10, D and E). We have reported that sensory nerves could detect bone density by a local "sensor," PGE2, released by osteoblasts, sensing internal organ signals for bone homeostasis (7). Moreover, the quantity of MSCs significantly decreased in sensory denervation mice, suggesting that sensory nerves function as a niche in the maintenance of MSCs. We have shown that sensory nerves transmit bone-forming signals by tuning down sympathetic nerve tone for osteoblast bone formation (7). In addition, we eliminated the possibility that osteocytic PGE2 might also be involved in sensory nerve regulation on MSCs (Supplemental Figure 12, $\mathrm{G}-\mathrm{L}$ ). Sympathetic tone is fulfilled by releasing norepinephrine and epinephrine, and an increase in epinephrine level could cause decreased bone formation $(47,48)$. Epinephrine has been shown to strongly induce adipogenic commitment of stem cells (49). Therefore, sensory nerves serve as a niche for MSCs, likely through control of epinephrine release of sympathetic tone. However, other sensory nerve secretory factors may also be involved in the maintenance of MSCs. Sensory nerves could also secrete neuropeptides, such as substance P, vasoactive intestinal peptide (50), and CGRP, which has been shown to promote osteoblast activity (51).

Deletion of the EP4 receptor in sensory nerves produced effects on bone formation and MSCs similar to those observed in the sensory denervation mouse model. EP4 belongs to the PGE2 receptor family, which consists of 4 receptors, EP1-EP4 (52). All 4
EPs have been globally knocked out, but only EP4-KO mice have a bone phenotype, providing evidence of a pivotal role of EP4 in PGE2-induced bone formation (53). Deletion of the EP4 receptor in osteoblast lineage cells did not result in a significant bone phenotype (54). Knockout of EP4 in sensory nerves increased adipogenesis and decreased osteogenesis and the ability of MSCs to differentiate into CFU-F. A role for EP4 in sensory nerve regulation of MSC differentiation indicates a possible mechanism for PGE2-induced bone formation. Moreover, mice with conditional knockout of EP4 in LepR ${ }^{+}$lineage cells had no bone phenotype and still positively responded to SW033921 treatment, which could potently stimulate bone formation (Supplemental Figure 13). Thus, PGE2 stimulates bone formation by activation of EP4 in sensory nerve regulation of MSCs. Concerning whether this regulatory axis is also functioning in other bones or metabolic systems, we did not find any effect of the PGE2/EP4 sensory nerve axis in mandible metabolism (Supplemental Figure 13); this may be due to the different MSC populations responsible for bone metabolism in different bones $(7,33,55)$. Interestingly, we found that $E P 4_{\text {Avil }}{ }^{-/}$mice had significant higher body fat mass compared with WT littermates (Supplemental Figure 14). This evidence indicates that PGE2/EP4 sensory nerve signaling has a potential impact on body fat and metabolic systems; the underlying mechanism will be a target of our future research.

CD $45^{-} \mathrm{CD} 31^{-} \mathrm{Sca}-1^{+} \mathrm{CD} 24^{+}$MSCs were used in our flow cytometry analysis (33). LepR has been reported to mark MSCs in adult mouse bone marrow, and LepR-Cre is effective for in vivo fate mapping of MSCs $(42,43)$. Nearly $13 \%$ of $\mathrm{LepR}^{+}$stromal cells are Sca- $1^{+}$, and all Sca- $1^{+}$stromal cells are $\mathrm{LepR}^{+}(42)$, which indicates that $\mathrm{LepR}^{+}$MSCs contain all CD $45^{-} \mathrm{CD} 31^{-} \mathrm{Sca}-1^{+} \mathrm{CD} 24^{+}$MSCs. Furthermore, LepR ${ }^{+}$stromal cells mark nearly $90 \%$ of bone marrow adipocytes in adult mice (42). Most recently, Baryawno et al. performed single-cell sequencing to build a bone marrow cellular taxonomy and showed that LepR ${ }^{+}$stromal cells formed a major cluster with the ability to partition into osteolineage and adipolineage cells (56). Therefore, LepR-Cre could effectively trace cells in both osteoblast and adipocyte lineages in fate mapping in a similar population of $\mathrm{CD} 45^{-} \mathrm{CD} 31^{-} \mathrm{Sca}-1^{+} \mathrm{CD} 24^{+}$MSCs. These results are consistent with our observation that sensory nerve regulation of bone homeostasis occurs only in adult mice, and not during developmental stages. The increase in adipogenesis after sensory denervation is also consistent with the high adipogenic potential of LepR $\mathrm{R}^{+}$MSCs. Indeed, our fate mapping result validates that increased adipocytes in sensory denervation mice were descendants of LepR ${ }^{+}$MSCs, considering that inhibition of sympathetic activity affected differentiation of $\mathrm{LepR}^{+}$MSCs in both sensory denervation mice and WT littermates. Sensory nerve regulation of bone homeostasis occurs primarily through LepR ${ }^{+}$MSCs in adult mouse bone marrow. In this regard, we do not exclude the involvement of other subgroups of MSCs such as Nestin ${ }^{+}$MSCs and PDG$\mathrm{F}^{+}$MSCs; however, nearly all $\mathrm{PDGF}^{+}$stromal cells are $\mathrm{LepR}^{+}$ MSCs, and vice versa, in mice aged 2-4 months (42), whereas Nestin $^{+}$MSCs are abundant in postnatal mice, and their frequency significantly decreases in adult mice (57).

Adipogenesis increased and osteogenesis decreased in $\mathrm{EP}_{\mathrm{Avil}^{-1}}$, $\operatorname{Trk} A_{A v i l}{ }^{-1-}$, and $C O X 2_{O C N}{ }^{-/-}$mice, but this phenotype could be caused by fate commitment of MSCs or alteration of progenitor cell prolif- 
eration. We have shown increased expression of adipogenic markers and decreased expression of osteogenic markers in uninduced MSCs in these knockout mice relative to control mice. Experiments showed that the frequency of $\mathrm{BrdU}^{+}$MSCs and $\mathrm{BrdU}^{+}$OPCs was significantly reduced in these knockout mice relative to control mice, while $\mathrm{BrdU}^{+} \mathrm{APC}$ were unaffected. These results suggest that sensory nerves are essential in the maintenance of self-renewal of MSCs. Sensory nerves also regulated the balance between osteogenic and adipogenic lineages in MSC fate determination. In particular, sensory nerves regulated the proliferation of OPCs, but not APCs, which is consistent with our previous finding that elevated sympathetic tone reduced the proliferation of osterix-positive osteoprogenitors (7). Therefore, these results reveal that sensory nerves regulate lineage commitment of MSCs and act as a niche for their maintenance, likely by regulating sympathetic tone.

\section{Methods}

Mice and in vivo treatment. The Advillin-Cre (Avil-Cre) mouse strain was provided by Xingzhong Dong (Department of Neuroscience, Johns Hopkins University). OCN-Cre mice were obtained from Thomas J. Clemens (Department of Orthopaedic Surgery, Johns Hopkins University). Trk $A^{f / / A}$ mice were obtained from David D. Ginty (Department of Neurobiology, Harvard Medical School, Boston, Massachusetts, USA). COX $2^{f / f l}$ mice were provided by Harvey Herschman (Department of Biological Chemistry, UCLA, Los Angeles, California, USA). EP4 ${ }^{f / f l}$ mice were obtained from Brian L. Kelsall (Laboratory of Molecular Immunology, NIH, Bethesda, Maryland, USA). iDTR ${ }^{f / l}$ mice and transgenic LepR-Cre mice, which expressed Cre recombinase under control of the mouse leptin receptor promoter, were purchased from the Jackson Laboratory $\left(i D T R^{f / / t}\right.$ stock no. 007900; LepR-Cre stock no. 008320). Heterozygous male Avil-Cre mice (female Avil-Cre mice were not used for breeding because of the risk of leakage of TrkA protein into the eggs) were crossed with a $\operatorname{Trk} A^{f / f l}, E P 4^{f / f l}$, or $i D T R^{f / f l}$ mouse. The offspring were intercrossed to generate the following genotypes:WT, Avil-Cre (Cre recombinase expression driven by Advillin promoter), Avil-Cre::EP4 $4^{A / f l}$ (conditional deletion of the EP4 receptor in Advillin lineage cells; referred to as EP4 ${ }_{A v i l}{ }^{-1}$ herein), Avil-Cre::TrkA $A^{f l f l}$ (referred to as TrkA $A_{A v i l}{ }^{-1}$ ), and Avil-Cre::iDTR ${ }^{f l++}$ (referred to as $\left.i D T R_{A v i}{ }^{+/}\right)$. To generate the inducible sensory denervation mouse model, we injected 8-week-old $i D T R_{\text {Avil }}{ }^{+/-}$mice with $1 \mu \mathrm{g} / \mathrm{kg}$ DTX 3 times a week for 4 consecutive weeks. Heterozygous $\mathrm{OCN}$-Cre mice were crossed with a $C O X 2^{f / f l}$ mouse; the offspring were intercrossed to generate the following genotypes: WT, OCN-Cre, and OCN-Cre::COX $2^{f / f t}$ (referred to as $\mathrm{COX} 2_{\mathrm{OCN}^{-}}^{- \text {) }}$. Heterozygous $\mathrm{OCN}$-Cre mice were crossed with an $E P 4^{f / f l}$ mouse, and the offspring were intercrossed to generate the following genotypes: WT (referred to as $E P 4^{f / f f}$ ) and $O C N-C r e:: E P 4^{f / f t}$ (conditional deletion of EP4 receptor in OCN lineage cells; referred to as $E P 4_{O C N}^{-1-}$ herein). LepR-Cre;YFP mice were crossed by LepR-Cre with Rosa26-YFP mice. The genotypes of the mice were determined by PCR analyses of genomic DNA, which was extracted from mouse tails with the following primers: Avil-Cre forward CCCTGTTCACTGTGAGTAGG, reverse GCGATCCCTGAACATGTCCATC; WT AGTATCTGGTAGGTGCTTCCAG; OCN-Cre forward CAAATAGCCCTGGCAGATTC, reverse TGATACAAGGGACATCTTCC; EP4 loxP allele forward TCTGTGAAGCGAGTCCTTAGGCT, reverse CGCACTCTCTCTCTCCCAAGGAA; COX2 loxP allele forward AATTACTGCTGAAGCCCACC, reverse GAATCTCCTAGAACTGACTGG; TrkA loxP allele forward AACAGTTTTGAGCATTTTCTATTGTTT, reverse CAAAGAAAACAGAAGAAAAATAATAC; IDTR loxP allele forward GCGAAGAGTTTGTCCTCAACC, reverse AAAGTCGCTCTGAGTTGTTAT. All animals were maintained at the animal facility of the Johns Hopkins University School of Medicine. We used all male mice in our experiments. We obtained whole blood samples by cardiac puncture immediately after euthanasia. Serum was collected by centrifuge at 200 $g$ for 15 minutes and stored at $-80^{\circ} \mathrm{C}$ before analyses. Femurs, tibias, and urine were also collected.

The drugs and compounds used in this study were as follows: DTX (MilliporeSigma, D0564), capsaicin (MilliporeSigma, M2028), propranolol (MilliporeSigma, 1576005), and SW033291 (Selleck, S7900). Dosages and time courses are noted in the corresponding text and figure legends.

$\mu C T$ analyses. Femurs were harvested from mice, and the soft tissue around the bone was removed, followed by fixation overnight using $4 \%$ paraformaldehyde. $\mu \mathrm{CT}$ analyses were performed by using a high-resolution $\mu \mathrm{CT}$ scanner (SkyScan, 1174). The scanning procedure was performed at $65 \mathrm{kV}$ with a $153-\mu \mathrm{A}$ current. Resolution was set to $8.7 \mu \mathrm{m} /$ pixel. Reconstruction software (NRecon, v1.6, SkyScan), data analysis software (CTAn, v1.9, SkyScan), and 3D model visualization software (CTVol, v2.0, SkyScan) were used to analyze the diaphyseal cortical and metaphyseal trabecular bone parameters of the femurs. We created cross-sectional images of the femur to perform 2D analyses of cortical bone and 3D analyses of trabecular bone. The region of interest of the trabecular bone was drawn beginning from $5 \%$ of the femur length proximal to the distal metaphyseal growth plate and extending proximally for another $5 \%$ of the total femur length. Trabecular BV/TV (Tb.BV/TV), Tb.Th, trabecular number (Tb.N), and trabecular separation (Tb.Sp) were collected from the 3D analysis data and used to represent the trabecular bone parameters.

$\mathrm{OsO}_{4}$ staining and $\mu \mathrm{CT}$ analysis. Femurs were harvested from mice, fixed in $4 \%$ phosphate-buffered paraformaldehyde for 48 hours, and decalcified for 2 weeks in $10 \%$ EDTA at $4^{\circ} \mathrm{C}$. The proximal ends of femurs were cut off and discarded. We incubated the distal part of femurs in $2 \%$ aqueous $\mathrm{OsO}_{4}$ (MilliporeSigma) for 2 hours in the fume hood. The femurs were rinsed in PBS for 48 hours and then scanned using a high-resolution $\mu$ CT scanner (SkyScan 1172, Bruker MicroCT) at $6-\mu \mathrm{m}$ resolution using 45 peak $\mathrm{kV}(\mathrm{kVp})$ and $177 \mu \mathrm{A}$. Quantification of adipocytes (Ad.N) and adipocyte volume/marrow volume (Ad.V/Ma.V) was registered to decalcified bone as previously described $(58,59)$.

Histology, immunohistochemistry, and immunofluorescence assay. Femurs were collected and fixed in $4 \%$ paraformaldehyde overnight and decalcified by using 10\% EDTA (pH 7.4) (AMRESCO, 0105) for 21 days. The samples were then dehydrated with $30 \%$ sucrose for 24 hours and embedded in paraffin or optimal cutting temperature compound (Sakura Finetek). We prepared $4-\mu \mathrm{m}$-thick coronal sections of the femur for SO/FG and Masson's staining according to the manufacturer's protocols. Briefly, sections were stained with Weigert's iron hematoxylin for 5 minutes, then counterstained with FG for 3 minutes, washed with $1 \%$ acetic acid, and stained in $0.1 \%$ SO. The sections were then dehydrated, clear mounted, and visualized by light microscopy; the cartilage matrix proteoglycans stained red, and bone compartments stained green. For Masson's staining, the sections were stained with Trichrome Stain (Masson) Kit (MilliporeSigma, HT15); cytoplasm and muscle fibers stained red; and bone tissue displayed blue coloration. Thick sections were cut as described previously (42). Briefly, the femurs were fixed for 4 hours with $4 \%$ paraformal- 
dehyde at $4^{\circ} \mathrm{C}$, then decalcified at $4^{\circ} \mathrm{C}$ using 0.5 M EDTA (pH 7.4) for 24 hours with constant shaking. The samples were dehydrated in $20 \%$ sucrose and $2 \%$ polyvinylpyrrolidone solution for 24 hours and embedded in $8 \%$ gelatin (MilliporeSigma, G1890) in the presence of $20 \%$ sucrose and $2 \%$ polyvinylpyrrolidone. Twenty- and $40-\mu \mathrm{m}$-thick coronal sections of the femurs were obtained, $20-\mu \mathrm{m}$-thick sections for femur adipocyte and osteoblast staining, and 40- $\mu \mathrm{m}$-thick sections for femur sensory nerve staining.

Immunostaining was performed using standard protocols. Briefly, the sections were incubated with primary antibodies against OCN (Takara Bio, M173, 1:200), perilipin (MilliporeSigma, P1873; 1:500), CGRP (Abcam, ab81887), GFP (Abcam, ab13970), and TrkA (R\&D Systems, AF1065) overnight at $4^{\circ} \mathrm{C}$. An HRP-streptavidin detection kit (Dako) was used in immunohistochemical procedures to detect immunoactivity, followed by counterstaining with hematoxylin (Dako, S3309). Fluorescence-conjugated secondary antibodies were used in immunofluorescence procedures to detect fluorescent signals after counterstaining with DAPI (Vector, H-1200). We used a Zeiss LSM 780 confocal microscope or an Olympus BX51 microscope for sample image capturing. A BrdU staining kit (Thermo Fisher Scientific, 88006599-45) was used to perform the BrdU immunostaining procedure. Quantitative histomorphometric analysis was performed by using OsteoMeasure XP Software (OsteoMetric) in a blinded fashion.

Flow cytometry assay. Mouse femurs were dissected, and soft tissue of the femur was removed. We then crushed femurs with sterilized bone scissors, and the bone pieces were further digested within a $10-\mathrm{mL}$ digesting buffer mixture of $\alpha$-MEM containing $3 \mathrm{mg} / \mathrm{mL}$ collagenase I (Worthington), $4 \mathrm{mg} / \mathrm{mL}$ dispase (MilliporeSigma), and $1 \mathrm{U} / \mathrm{mL}$ DNAse I (Invitrogen) for 20 minutes in a shaking water bath at $37^{\circ} \mathrm{C}$. The suspension was passed through a $70-\mu \mathrm{m}$ cell strainer to remove tissue fragments and centrifuged at $300 \mathrm{~g}$ for 5 minutes at $4^{\circ} \mathrm{C}$. The pellet was resuspended in ACK lysing buffer (BD Biosciences) to exclude red blood cells and centrifuged at $300 \mathrm{~g}$ for 5 minutes at $4^{\circ} \mathrm{C}$. The pellet was resuspended in $100 \mu \mathrm{L}$ staining buffer (BioLegend) and stained with antibodies for 30 minutes at $4^{\circ} \mathrm{C}$. The antibodies were as follows: anti-CD31-Brilliant Violet 421 (BioLegend, 102424; 1:200), anti-CD45-Brilliant Violet 421 (BioLegend, 103134; 1:200), antiTer119-Brilliant Violet 421 (BioLegend, 116234; 1:200), anti-mouse Sca-1-APC/CY7 (BioLegend, 108126; 1:200), anti-CD24-PE (eBioscience, clone 30-F1, 1920468), and anti-mouse CD140a (eBioscience, 135908). The dead cells were marked by using a fixable dead cell stain kit (Molecular Probes), and living cells were gated for lack of UV fluorescence. Before flow cytometry, cells were resuspended in staining buffer and analyzed on an LSR II flow cytometer (BD Biosciences).

$C F U-F, C F U-O B$, and $C F U-A D$ in vitro differentiation assays. Bone marrow digestion and CFU-F, CFU-OB and CFU-AD assays of mouse bone marrow cells were based on previously described protocols with customization $(38,39)$. Briefly, for CFU assays with unfractionated bone marrow cells, freshly isolated single-cell suspensions from femur of 12-week-old male mice were plated at a density of $5 \times 10^{4} / \mathrm{cm}^{2}$ in 6-well plates in DMEM (Gibco) with 15\% FBS (Gibco), $10 \mu \mathrm{mol} / \mathrm{L}$ Y-27632 (STEMCELL Technologies), and 1\% penicillin/streptomycin (MilliporeSigma) and incubated at $37^{\circ} \mathrm{C}$. For CFU-F assays with sorted cells, cells were sorted into culture at a density of $10 / \mathrm{cm}^{2}$ in 6-well plates, ensuring that colonies would form at clonal density to allow counting; CFU-F colonies were counted with crystal violet staining after 10 days of expansion, and we included the colonies that con- tained 50 cells or more. For the in vitro osteoblast differentiation assays (CFU-OB), cells were seeded at a density of $5 \times 10^{3} / \mathrm{cm}^{2}$ and stained with alizarin red (MilliporeSigma) after 21 days of culture with StemPro Osteogenesis Differentiation Kits (Gibco). For in vitro adipocyte differentiation assays (CFU-AD), cells were seeded at a density of $1 \times 10^{4} / \mathrm{cm}^{2}$ and stained with oil red O (MilliporeSigma) after 14 days of culture with a StemPro Adipogenesis Differentiation Kit (Gibco).

qPCR. Total RNA was purified from cells in culture or tissues using TRIzol (Invitrogen, 15596026), following the manufacturer's protocol. We performed qPCR using the SYBR Green Power PCR Master Mix (Invitrogen, A25777) on a CFX Connect instrument (Bio-Rad); Gapdh amplification was used as an internal control. Dissociation curve analysis was performed for every experiment. Sequences of the primers used for each gene were as follows: Pparg forward ACCACTCGCATTCCTTTGAC, reverse TGGGTCAGCTCTTGTGAATG; Cebpa forward AAACAACGCAACGTGGAGA, reverse GCGGTCATTGTCACTGGTC; Fabp 4 forward CATCAGCGTAAATGGGGATT, reverse GTCGTCTGCGGTGATTTCAT; Alp forward ATCTTTGGTCTGGCTCCCATG, reverse TGAGCGACACGGACAAGAAGCCCTT; Col1a1 forward GACGCCATCAAGGTCTACTG, reverse ACGGGAATCCATCGGTCA; Runx2 forward TTACCTACACCCCGCCAGTC, reverse TGCTGGTCTGGAAGGGTCC.

In vivo BrdU incorporation assay. The BrdU assay was conducted as previously described (42). Briefly, mice were injected intraperitoneally with a single dose of $100 \mathrm{mg} / \mathrm{kg}$ BrdU (MilliporeSigma) diluted in sterilized PBS. The effect of BrdU labeling was maintained by giving BrdU via drinking water at a concentration of $0.5 \mathrm{mg} / \mathrm{mL}$. Drinking water was renewed every other day. For BrdU analysis in flow cytometry, we used an APC-BrdU flow kit (BD Bioscience) according to the manufacturer's instructions.

Bone regeneration and fracture models. Mice underwent general anesthesia. The bone regeneration model was established as described previously (5). Briefly, a longitudinal incision was made on each knee to expose the femoral condyle by patella dislocation. Then, a hole was made at the intercondylar notch of the femur using a dental drill. A 0.6-mm-diameter Kirschner wire was placed from the proximal end of the femur to confirm marrow ablation by radiography. The dislocated patella was reposed, and the skin was sutured after removal of the Kirschner wire. Bone samples were harvested 7 days after bone marrow ablation, as described above.

The bone fracture procedure was performed as described previously (51). Briefly, after mice were anesthetized, a stainless steel pin was inserted into the intramedullary canal from the distal femur to stabilize the fracture region. The pin was fixed in place by a wedge that was made by bending the first $2 \mathrm{~mm}$ of a 30-gauge needle. Fracture was made by 3 -point bending, and the surgery region was then sutured. The treated mice were transferred into cages when they recovered from surgery and were checked twice a day. The fractured femurs were harvested, and the pin was removed 2 weeks postoperatively. $\mu \mathrm{CT}$, bone sectioning, and staining were further performed on these bone samples.

Statistics. All data analyses were performed using SPSS software, version 15.0 (IBM). Data are presented as mean \pm SEM. For comparisons between 2 groups, we used 2-tailed Student's $t$ tests. For comparisons among multiple groups, we used 1-way ANOVA. A $P$ value less than 0.05 was deemed significant. All representative experiments were repeated at least 3 times. All relevant data are available from the 
authors. Methods for the Von Frey test, hot plate test, kidney transplantation, metabolic studies, and ELISA assay are described in Supplemental Methods.

Study approval. All animal experiments were performed in accordance with NIH policies on the use of laboratory animals. All experimental protocols were approved by the Animal Care and Use Committee of Johns Hopkins University.

\section{Author contributions}

$\mathrm{BH}$ and $\mathrm{X}$. Lv performed most of the experiments. Hao Chen, PX, MY, BG, and GZ helped with mouse breeding and genotyping. PW and DP analyzed the $\mu \mathrm{CT}$ data. X. Liu, ZL, and JLC helped with flow cytometry analysis. WS, XW, and SL performed the statistical analysis. RD, SN, YL, and LW helped with mouse euthanization and sample collections. ZS, YZ, and Huajiang Chen offered equipment and valuable discussion. MW and WY read and revised the manuscript. XC conceived the study and wrote the manuscript.
The authorship order among co-first authors was determined by alphabetical sequence.

\section{Acknowledgments}

This research was supported by NIH grant AR 071432 (to XC). We thank editors Jenni Weems and Rachel Box in the editorial office at the Department of Orthopaedic Surgery at Johns Hopkins University for editing the manuscript.

Address correspondence to: Xu Cao, Department of Orthopaedic Surgery, Johns Hopkins University, 720 Rutland Avenue, Baltimore, Maryland 21205, USA. Phone: 205.643.4333, Email: xcao11@jhmi.edu. Or to: Wen Yuan, Section of Spine Surgery, Department of Orthopaedics, Changzheng Hospital, Second Military Medical University, 415 Fengyang Road, Huangpu District, Shanghai, China. Phone: 86.21.81885630, Email: yuanwenspine@126.com.
1. Snyder DJ, Bartoshuk LM. Oral sensory nerve damage: Causes and consequences. Rev Endocr Metab Disord. 2016;17(2):149-158.

2. Pinho-Ribeiro FA, Verri WA, Chiu IM. Nociceptor sensory neuron-immune interactions in pain and inflammation. Trends Immunol. 2017;38(1):5-19.

3. Delmas P, Hao J, Rodat-Despoix L. Molecular mechanisms of mechanotransduction in mammalian sensory neurons. Nat Rev Neurosci. 2011;12(3):139-153.

4. Jung WC, Levesque JP, Ruitenberg MJ. It takes nerve to fight back: the significance of neural innervation of the bone marrow and spleen for immune function. Semin Cell Dev Biol. 2017;61:60-70.

5. Fukuda T, et al. Sema3A regulates bone-mass accrual through sensory innervations. Nature. 2013;497(7450):490-493.

6. Takeda S, et al. Leptin regulates bone formation via the sympathetic nervous system. Cell. 2002;111(3):305-317.

7. Chen $\mathrm{H}$, et al. Prostaglandin E2 mediates sensory nerve regulation of bone homeostasis. Nat Commun. 2019;10(1):181.

8. Blackwell KA, Raisz LG, Pilbeam CC. Prostaglandins in bone: bad cop, good cop? Trends Endocrinol Metab. 2010;21(5):294-301.

9. Uppal S, et al. Mutations in 15-hydroxyprostaglandin dehydrogenase cause primary hypertrophic osteoarthropathy. Nat Genet. 2008;40(6):789-793.

10. Kawabata A. Prostaglandin E2 and pain - an update. Biol Pharm Bull. 2011;34(8):1170-1173.

11. Kuner R. Central mechanisms of pathological pain. Nat Med. 2010;16(11):1258-1266.

12. Zhang Y, et al. Tissue regeneration. Inhibition of the prostaglandin-degrading enzyme 15-PGDH potentiates tissue regeneration. Science. 2015;348(6240):aaa2340.

13. Minamizaki T, Yoshiko Y, Kozai K, Aubin JE, Maeda N. EP2 and EP4 receptors differentially mediate MAPK pathways underlying anabolic actions of prostaglandin $\mathrm{E} 2$ on bone formation in rat calvaria cell cultures. Bone. 2009;44(6):1177-1185.

14. Gao Q, Xu M, Alander CB, Choudhary S, Pilbeam CC, Raisz LG. Effects of prostaglandin E2 on bone in mice in vivo. Prostaglandins Other Lipid Mediat. 2009;89(1-2):20-25.

15. Crane JL, Cao X. Bone marrow mesenchymal stem cells and TGF- $\beta$ signaling in bone remodeling. JClin Invest. 2014;124(2):466-472.

16. Zhen $G$, et al. Inhibition of TGF- $\beta$ signaling in mesenchymal stem cells of subchondral bone attenuates osteoarthritis. Nat Med. 2013;19(6):704-712.

17. Tang Y, et al. TGF-beta1-induced migration of bone mesenchymal stem cells couples bone resorption with formation. Nat Med. 2009;15(7):757-765.

18. Xie H, et al. PDGF-BB secreted by preosteoclasts induces angiogenesis during coupling with osteogenesis. Nat Med. 2014;20(11):1270-1278.

19. Uccelli A, Moretta L, Pistoia V. Mesenchymal stem cells in health and disease. Nat Rev Immu nol. 2008;8(9):726-736.

20. Ellies DL, Krumlauf R. Bone formation: the nuclear matrix reloaded. Cell. 2006;125(5):840-842.

21. Fridenshteĭn AIa, Petrakova KV, Kuralesova AI, Frolova GI. [Precursor cells for osteogenic and hemopoietic tissues. Analysis of heterotopic transplants of bone marrow]. Tsitologiia. 1968;10(5):557-567.

22. Bianco P. Bone and the hematopoietic niche: a tale of two stem cells. Blood. 2011;117(20):5281-5288.

23. Park D, et al. Endogenous bone marrow MSCs are dynamic, fate-restricted participants in bone maintenance and regeneration. Cell Stem Cell. 2012;10(3):259-272.

24. Mendelson A, Frenette PS. Hematopoietic stem cell niche maintenance during homeostasis and regeneration. Nat Med. 2014;20(8):833-846.

25. Morrison SJ, Scadden DT. The bone marrow niche for haematopoietic stem cells. Nature. 2014;505(7483):327-334.

26. Prockop DJ. Marrow stromal cells as stem cells for nonhematopoietic tissues. Science. 1997;276(5309):71-74.

27. Méndez-Ferrer S, et al. Mesenchymal and haematopoietic stem cells form a unique bone marrow niche. Nature. 2010;466(7308):829-834.

28. Schneider RK, et al. Gli1 ${ }^{+}$mesenchymal stromal cells are a key driver of bone marrow fibrosis and an important cellular therapeutic target. Cell Stem Cell. 2018;23(2):308-309.

29. Decker M, et al. Leptin-receptor-expressing bone marrow stromal cells are myofibroblasts in primary myelofibrosis. Nat Cell Biol. 2017;19(6):677-688.

30. Abbuehl JP, Tatarova Z, Held W, Huelsken J. Long-term engraftment of primary bone marrow stromal cells repairs niche damage and improves hematopoietic stem cell transplantation. Cell Stem Cell. 2017;21(2):241-255.e6.

31. Maryanovich M, et al. Adrenergic nerve degeneration in bone marrow drives aging of the hematopoietic stem cell niche. Nat Med. 2018;24(6):782-791.

32. Katayama Y, et al. Signals from the sympathetic nervous system regulate hematopoietic stem cell egress from bone marrow. Cell. 2006;124(2):407-421.

33. Ambrosi TH, et al. Adipocyte accumulation in the bone marrow during obesity and aging impairs stem cell-based hematopoietic and bone regeneration. Cell Stem Cell. 2017;20(6):771-784.e6.

34. Guan M, et al. Directing mesenchymal stem cells to bone to augment bone formation and increase bone mass. Nat Med. 2012;18(3):456-462.

35. Worthley DL, et al. Gremlin 1 identifies a skeletal stem cell with bone, cartilage, and reticular stromal potential. Cell. 2015;160(1-2):269-284.

36. Chan CK, et al. Identification and specification of the mouse skeletal stem cell. Cell. 2015;160(1-2):285-298.

37. Yu B, et al. PGC-1 $\alpha$ controls skeletal stem cell fate and bone-fat balance in osteoporosis and skeletal aging by inducing TAZ. Cell Stem Cell. 2018;23(2):193-209.e5.

38. Chan CKF, et al. Identification of the human skeletal stem cell. Cell. 2018;175(1):43-56.e21.

39. Wu X, et al. Inhibition of Sca-1-positive skeletal stem cell recruitment by alendronate blunts the anabolic effects of parathyroid hormone on bone remodeling. Cell Stem Cell. 2010;7(5):571-580.

40. Mizuhashi K, et al. Resting zone of the growth plate houses a unique class of skeletal stem cells. Nature. 2018;563(7730):254-258.

41. Fan Y, et al. Parathyroid hormone directs bone marrow mesenchymal cell fate. Cell Metab. 
2017;25(3):661-672.

42. Zhou BO, Yue R, Murphy MM, Peyer JG, Morrison SJ. Leptin-receptor-expressing mesenchymal stromal cells represent the main source of bone formed by adult bone marrow. Cell Stem Cell. 2014;15(2):154-168.

43. Yue R, Zhou BO, Shimada IS, Zhao Z, Morrison SJ. Leptin receptor promotes adipogenesis and reduces osteogenesis by regulating mesenchymal stromal cells in adult bone marrow. Cell Stem Cell. 2016;18(6):782-796.

44. Shi Y, He G, Lee WC, McKenzie JA, Silva MJ, Long F. Gli1 identifies osteogenic progenitors for bone formation and fracture repair. Nat Commun. 2017;8(1):2043.

45. Mizoguchi T, et al. Osterix marks distinct waves of primitive and definitive stromal progenitors during bone marrow development. Dev Cell. 2014;29(3):340-349.

46. Kfoury Y, Scadden DT. Mesenchymal cell contributions to the stem cell niche. Cell Stem Cell. 2015;16(3):239-253.

47. Ortuño MJ, et al. Serotonin-reuptake inhibitors act centrally to cause bone loss in mice by counteracting a local anti-resorptive effect. Nat Med. 2016;22(10):1170-1179.

48. Elefteriou F, et al. Leptin regulation of bone resorption by the sympathetic nervous system and CART. Nature. 2005;434(7032):514-520.

49. Han R, Kitlinska JB, Munday WR, Gallicano GI, Zukowska Z. Stress hormone epinephrine enhances adipogenesis in murine embryonic stem cells by up-regulating the neuropeptide $\mathrm{Y}$ system. Plos One. 2012;7(5):e36609.

50. Riesco N, Cernuda-Morollón E, Pascual J. Neuropeptides as a marker for chronic headache. Curr Pain Headache Rep. 2017;21(4):18.

51. Zhang Y, et al. Implant-derived magnesium induces local neuronal production of CGRP to improve bone-fracture healing in rats. Nat Med. 2016;22(10):1160-1169.

52. Furuyashiki T, Narumiya S. Stress responses: the contribution of prostaglandin $\mathrm{E}(2)$ and its receptors. Nat Rev Endocrinol. 2011;7(3):163-175.

53. Yoshida K, et al. Stimulation of bone formation and prevention of bone loss by prostaglandin $\mathrm{E}$
EP4 receptor activation. Proc Natl Acad Sci U S A. 2002;99(7):4580-4585.

54. Gao Q, et al. Effects of global or targeted deletion of the EP4 receptor on the response of osteoblasts to prostaglandin in vitro and on bone histomorphometry in aged mice. Bone. 2009;45(1):98-103.

55. Zhao H, Feng J, Ho TV, Grimes W, Urata M, Chai $\mathrm{Y}$. The suture provides a niche for mesenchymal stem cells of craniofacial bones. Nat Cell Biol. 2015;17(4):386-396.

56. Baryawno N, et al. A cellular taxonomy of the bone marrow stroma in homeostasis and leukemia. Cell. 2019;177(7):1915-1932.e16.

57. Kunisaki $Y$, et al. Arteriolar niches maintain haematopoietic stem cell quiescence. Nature. 2013;502(7473):637-643.

58. Li CJ, et al. Long noncoding RNA Bmncr regulates mesenchymal stem cell fate during skeletal aging. J Clin Invest. 2018;128(12):5251-5266.

59. Xiao Z, et al. Osteoblast-specific deletion of Pkd2 leads to low-turnover osteopenia and reduced bone marrow adiposity. PLoS One. 2014;9(12):e114198. 